\title{
Equivalence of non-equilibrium ensembles and representation of friction in turbulent flows: the Lorenz 96 model
}

Article

Accepted Version

Gallavotti, G. and Lucarini, V. (2014) Equivalence of nonequilibrium ensembles and representation of friction in turbulent flows: the Lorenz 96 model. Journal of Statistical Physics, 156 (6). pp. 1027-1065. ISSN 0022-4715 doi: https://doi.org/10.1007/s10955-014-1051-6 Available at https://centaur.reading.ac.uk/67610/

It is advisable to refer to the publisher's version if you intend to cite from the work. See Guidance on citing.

To link to this article DOI: http://dx.doi.org/10.1007/s10955-014-1051-6

Publisher: Springer

All outputs in CentAUR are protected by Intellectual Property Rights law, including copyright law. Copyright and IPR is retained by the creators or other copyright holders. Terms and conditions for use of this material are defined in the End User Agreement.

www.reading.ac.uk/centaur 
Central Archive at the University of Reading

Reading's research outputs online 


\title{
Equivalence of Non-Equilibrium Ensembles and Representation of Friction in Turbulent Flows: The Lorenz 96 Model
}

\author{
Giovanni Gallavotti \\ Department of Physics, University of Rome La Sapienza \\ Department of Mathematics, Rutgers University, Rutgers, USA \\ Valerio Lucarini \\ Institute of Meteorology, Klimacampus, University of Hamburg,Hamburg, Germany \\ Department of Mathematics and Statistics, University of Reading, Reading, UK \\ Walker Institute for Climate Change Research, University of Reading, Reading, UK \\ August 4, 2017
}

\begin{abstract}
We construct different equivalent non-equilibrium statistical ensembles in a simple yet instructive $N$-degrees of freedom model of atmospheric turbulence, introduced by Lorenz in 1996. The vector field can be decomposed into an energy-conserving, time-reversible part, plus a non-time reversible part, including forcing and dissipation. We construct a modified version of the model where viscosity varies with time, in such a way that energy is conserved, and the resulting dynamics is fully time-reversible. For each value of the forcing, the statistical properties of the irreversible and reversible model are in excellent agreement, if in the latter the energy is kept constant at a value equal to the time-average realized with the irreversible model. In particular, the average contraction rate of the phase space of the time-reversible model agrees with that of the irreversible model, where instead it is constant by construction. We also show that the phase space contraction rate obeys the fluctuation relation, and we relate its finite time corrections to the characteristic time scales of the system. A local version of the fluctuation relation is explored and successfully checked. The equivalence between the two non-equilibrium ensembles extends to dynamical properties such as the Lyapunov exponents, which are shown to obey to a good degree of approximation a pairing rule. These results have relevance in motivating the importance of the chaotic hypothesis. in explaining that we have the freedom to model non-equilibrium systems using different but equivalent approaches, and, in particular, that using a model of a fluid where viscosity is kept constant is just one option, and not necessarily the only option, for describing accurately its statistical and dynamical properties.
\end{abstract}

Key words: Equivalent Equations, Turbulence, Chaotic Hypothesis, Fluctuation Theorem, Geophysical Flows, Parametrization, Lyapunov Exponents 


\section{Introduction}

Non-equilibrium statistical mechanical systems reach a steady state, after transients have died out, with a statistical balance between forcing and dissipation. The forcing is typically performed by an external field, while the dissipation is taken care of by a suitably defined thermostat, which has the role of removing the excess of energy accumulated in the system as a result of the work due to the external field and the related processes cascading from there.

When considering a macroscopic description of a physical system, friction plays the role of the thermostat. The most common way of introducing it is phenomelogically described by a force opposing motion proportional to a friction constant $\nu$. The introduction of friction in this form leads to a fundamental change in the equation of motion of the system, as the time reversal symmetry is broken. This cannot be a fundamental model of the process of thermostatting because the basic equations of Physics are time reversal invariant, i.e. invariant under a transformation $I$ which anticommutes with the time evolution map $x \rightarrow S_{t} x$, in the sense that $S_{t} I x=I S_{-t} x$ (which usually is just a velocity reversal, but might be more involved, [12].

Hence, it is worth investigating whether the statistical, macroscopic properties of a physical system whose microscopic dynamics obeys a simple phenomenological friction law of the kind described above can be equivalently described by different microscopic equations, constructed in such a way that fundamental time reversal symmetry is preserved [11, 10, 14]. The main reason for studying this is not the hope for simpler equations, but rather the possibility of having an alternative view of the dynamics, which could reveal certain features of the problem.

A compelling analogy can be found with equilibrium statistical mechanics, and in particular with the concept of equivalence of the ensembles, which suggests, e.g. that the same system can be equivalently described by the microcanonical or canonical ensembles. Obviously, the equivalence does not extend to all properties: If one is interested in energy (temperature) fluctuations, it would be futile, by construction, to use the microcanonical (canonical) ensemble. A similar discussion has not yet been systematically carried over in the context of nonequilibrium. An early attempt at a check of the ideas in the works cited above can be found in [21]. For instance if interest focuses on dissipation fluctuations a model in which the dissipation is a fixed constant may affect deeply dissipation itself (obviously), and other observables.

Since reasonable Physics will not dismiss dissipative equations, like the 
Navier-Stokes (NS) equations, the approach that should be taken is to regard the stationary states reached in models with equations in which friction is constant as ensembles whose properties can also be described by equivalent ensembles, which are stationary states of other equations. In particular, our goal is to construct such ensembles from evolution equations obeying time reversal symmetry.

In this paper a detailed investigation is pursued on a specific model and the result suggest a very general picture, whose foundation was laid out in [11, 10]. The conceptual frame is the chaotic hypothesis which leads to the emergence of various properties making use of the general theory of chaotic motions initiated in [25, 38] and allows deriving parameter free predictions on various fluctuations like the fluctuation theorem [19].

It is important to keep in mind that the representation of dissipative processes in e.g. fluids, except in the rare cases where direct numerical simulation down to the Kolmogorov scale is feasible and one can use molecular diffusivities and viscosities, requires considerable theoretical efforts. In fact, one must find simplified yet accurate methods for dealing with the unresolved scales of motion and for representing correctly complex cascades of quantities like energy and enstrophy. The formulation of methods for performing large eddy simulations [42, 39, 7], and, more in general, for providing a closure to Reynolds stresses [33] provide prototypical examples in this direction. The aim of our work is, however, to study in a simple example the fundamental role of time reversibility in dissipative phenomena, and shows, at least in the model considered, how to avoid using equations breaking time reversal invariance without loss of information. Hence it addresses specifically time reversal symmetry (and possibly other symmetry properties), unlike the previous literature in the field, as the just works cited above, which model dissipation phenomenologically with irreversible equations. A notable exception to this approach in the existing literature can be found in [40] where it is shown, for the first time, that is possible to reproduce the properties of a dynamical system where a constant friction plays a key role using a model obeying time reversibility.

\subsection{Equivalence of Ensembles}

Let us consider a dynamical system with $N$ degrees of freedom

$$
\dot{x}_{j}=f_{j}(x)+F_{j}-\nu(L x)_{j}, \quad \nu>0, j=1, \ldots, N
$$

where $L$ is a positive definite dissipation matrix: e.g. in many interesting cases and $(L x)_{j}=x_{j}$ and $\nu>0$, and $f(x)=-f(-x)$ (time reversibility). 
Let $E(x)$ be an observable such that $\sum_{j=1}^{N} \partial_{j} E(x)(L x)_{j}=M(x)$ is positive for $x \neq 0$. In the simple case where $L$ is the identity, taking $E(x)=\frac{1}{2} \sum_{j} x_{j}^{2} \equiv x^{2}$, we have $M(x)=x^{2}$; then the equation

$$
\dot{x}_{j}=f_{j}(x)+F_{j}-\alpha(x)(L x)_{j}, \quad \alpha(x) \stackrel{\operatorname{def}}{=} \frac{\sum_{j=1}^{N} F_{j} \partial_{j} E}{M(x)},
$$

will admit $E(x)$ as an exact constant of motion, and, if $E(x)=E(-x)$, it will be time-reversible with time reversal $x \rightarrow-x, t \rightarrow-t$. The quantity $\alpha(x)$ will fluctuate in time and, in general, will not have a definite sign. We say that the stationary distributions of the Eq.11.1, 1.2 define corresponding ensembles of statistical distributions.

A natural generalization to cases with several friction constants is obtained if $L_{\boldsymbol{\nu}}$ is a positive matrix depending linearly on $k$ positive friction constants $\boldsymbol{\nu}=\left(\nu_{1}, \ldots, \nu_{k}\right),\left(L_{\boldsymbol{\nu}} x\right)_{j}=\sum_{j^{\prime}=1}^{N} \sum_{s=1}^{k} \nu_{s} L_{j, j^{\prime}}^{s} x_{j^{\prime}}$ : then the stationary distributions for the equations

$$
\dot{x}_{j}=f_{j}(x)+F_{j}-\left(L_{\boldsymbol{\nu}} x\right)_{j}, \quad \text { and } \quad \dot{x}_{j}=f_{j}(x)+F_{j}-\left(L_{\boldsymbol{\alpha}} x\right)_{j},
$$

will be said to form two corresponding ensembles of statistical distributions with dissipation balanced on $k$ observables $\mathbf{E}(x)=\left(E_{1}(x), \ldots, E_{k}(x)\right)$, with $E_{j}(x)=E_{j}(-x)$, if the $k \times k$ matrices

$$
M_{r, s}(x) \stackrel{\text { def }}{=} \sum_{j, j^{\prime}=1}^{N} \partial_{j} E_{r}(x) L_{j, j^{\prime}}^{s} x_{j^{\prime}}
$$

are positive definite for $x \neq 0$, and if the coefficients $\boldsymbol{\alpha}(x)=\left(\alpha_{1}(x), \ldots, \alpha_{k}(x)\right)$ are defined as

$$
\begin{aligned}
& \alpha_{r}(x)=\sum_{s=1}^{k} M(x)_{r s}^{-1} \sum_{j=1}^{N} \partial_{j} E_{s}(x) f_{j}(x), \\
& \boldsymbol{\alpha}=M(x)^{-1} \boldsymbol{\Phi}(x), \quad \boldsymbol{\Phi}_{r}(x)=\left(\mathbf{F}(x) \cdot \boldsymbol{\partial} E_{r}(x)\right)
\end{aligned}
$$

The key property of the equations $(1.5)$ is that $\mathbf{E}(x)$ are $k$ exact constants of motion; furthermore the equation is time reversal invariant (with time reversal symmetry given by $I: x \rightarrow-x, t \rightarrow-t$ ), as opposed to Eq.(1.1).

The equations Eq. (1.3) will be said to have 'dissipation balanced on the observables $\mathbf{E}$ '.

A simple class of equations with dissipation balanced on $\mathbf{E}(x)$ is obtained in the cases in which $\mathbf{E}(x)$ are quadratic positive definite constants of motion for the inviscid, not forced equation $\dot{x}=f(x)$ and the viscous equations are 


$$
\dot{x}_{j}=f_{j}(x)+F_{j}(x)-\sum_{r=1}^{k} \nu_{r} \partial_{j} E_{r}(x)
$$

In this case the $\boldsymbol{\Phi}(x)$ does not depend explicitly on $f$. However the balanced dissipation equations are more general.

In [11, 15], it was proposed, in special cases and for strong forcing, that given a dissipative equation, the constant friction vector $\boldsymbol{\nu}$ could be replaced by the corresponding time reversible equations with a variable vector $\boldsymbol{\alpha}=\left(\alpha_{1}, \ldots, \alpha_{k}\right)$ as defined above which obeys time reversal symmetry, as opposed to the original dissipative-balanced system.

As a notable example, the incompressible 2-dimensional Navier-Stokes (NS) equation in a periodic container can interpreted, according to Eq.(1.1), as describing a dissipative-balanced system:

$$
\dot{\mathbf{u}}+(\mathbf{u} \cdot \boldsymbol{\partial}) \mathbf{u}=-\boldsymbol{\partial} p+\mathbf{g}+\nu \Delta \mathbf{u}=0, \quad \boldsymbol{\partial} \cdot \mathbf{u}=0
$$

because $E(\mathbf{u})=\frac{1}{2} \int(\boldsymbol{\partial u})^{2}(x) d x$, being the dissipation of kinetic energy (apart from a factor $1 / \nu$ ), is a constant of motion in $2 D$ incompressible fluids with periodic boundary conditions and $\frac{\delta E(\mathbf{u})}{\delta u_{j}(x)}=\int \sum_{r k} \partial_{r} u_{k} \delta_{j k} \partial_{r} \delta(y-$ $x) d x \equiv-\Delta u_{j}(x)$. The equivalent model is then:

$$
\begin{gathered}
\dot{\mathbf{u}}+(\mathbf{u} \cdot \boldsymbol{\partial}) \mathbf{u}=-\boldsymbol{\partial} p+\mathbf{g}+\alpha(\mathbf{u}) \Delta \mathbf{u}, \quad \boldsymbol{\partial} \cdot \mathbf{u}=0 \\
\alpha(\mathbf{u}) \stackrel{\text { def }}{=} \frac{-\int \mathbf{g} \cdot \Delta \mathbf{u} d \mathbf{x}+\int \Delta \mathbf{u} \cdot((\underline{u} \cdot \underset{\sim}{\partial}) \mathbf{u}) d \mathbf{x}}{\int(\Delta \mathbf{u})^{2}}
\end{gathered}
$$

which via the spatial Fourier decomposition of the velocity field $\mathbf{u}$ becomes:

$$
\alpha(\mathbf{u}) \stackrel{\text { def }}{=} \frac{\sum_{\mathbf{k}} \mathbf{k}^{2} \mathbf{g}_{\mathbf{k}} \cdot \mathbf{u}_{-\mathbf{k}}}{\sum_{\mathbf{k}} \mathbf{k}^{4}\left|\mathbf{u}_{\mathbf{k}}\right|^{2}}, \quad D=2
$$

In dimension 3 the same fluid with dissipation balanced on the vorticity observable $E(\mathbf{u})$ cannot be regarded as an element of the special class given in Eq. 1.6 where the observables $\mathbf{E}$ are constants of motion and the viscous term contains their derivatives, because in this case $E(\mathbf{u})$ in absence of friction $E(\mathbf{u})$ is not conserved. If one considers instead as conserved quantity for the unviscid flow $K(\mathbf{u})=\int \mathbf{u}(x)^{2} d x$, the corresponding irreversible equations (both in 2 and 3 dimensions) of the form given in Eq. 1.6 are the the so-called Ekman friction equations, where the dissipative term $\Delta \mathbf{u}$ of the Navier-Stokes equations is substituted by a term of the form $-\mathbf{u}$. 
The $3 D$ fluid can nevertheless be considered to have dissipation balanced on the vorticity as in Eq. (1.8): in this case the inertial term will appear explicitly in the definition of $\alpha(x)$, so that $\alpha(x)$ will be given by Eq. 1.8 and it will not be as simple as Eq.(1.9). It will be

$$
\alpha(\mathbf{u}) \stackrel{\text { def }}{=} \frac{\sum_{\mathbf{k}} \mathbf{k}^{2} \mathbf{g}_{\mathbf{k}} \cdot \mathbf{u}_{-\mathbf{k}}+\mathbf{k}^{2} \mathbf{u}_{-\mathbf{k}} \cdot \sum_{\mathbf{h}}(\underline{u} \mathbf{k}-\mathbf{h} \cdot i \underset{\sim}{h}) \mathbf{u}_{\mathbf{h}}}{\sum_{\mathbf{k}} \mathbf{k}^{4}\left|\mathbf{u}_{\mathbf{k}}\right|^{2}}, \quad D=3
$$

The equations Eq.(1.8), (1.9) have the property of having $E_{j}(x) \forall j=1, \ldots, k$, or, respectively, $E(\mathbf{u})$, exactly constant.

The proposed equivalence between the balanced dissipative equations and the corresponding reversible equations is articulated in several properties of their stationary distributions, reminiscent of the equivalence statements between different ensembles in statistical mechanics.

Let $\mu_{\boldsymbol{\nu}}^{i}$ be the stationary distribution for the irreversible balanced dissipative system with friction coefficients $\nu$ and $\mu_{\mathbf{E}}^{r}$ the stationary distribution for the reversible equation with $\mathbf{E}$ as a constant of motion vector with value $\overline{\mathbf{E}}$. Then $\mu_{\boldsymbol{\nu}}^{i}$ will be said to correspond to $\mu_{\overline{\mathbf{E}}}^{r}$ if $\mu_{\boldsymbol{\nu}}^{i}(\mathbf{E})=\overline{\mathbf{E}}$ or (equivalently, as we shall argue) if $\mu_{\mathbf{E}}^{r}(\boldsymbol{\alpha})=\boldsymbol{\nu}$.

The correspondence that we discuss will be interpreted to imply equivalence: i.e. that setting the value of the viscosity coefficient $\boldsymbol{\nu}$ is conceptually equivalent to setting the value of the physical quantities $\mathbf{E}$, at least in the limit of large forcing. Therefore, it will become possible, at least in the cases and under the assumptions discussed in the following, to take two different, but equally effective, points of view for studying the properties of the considered system in the same sense in which different equilibrium ensembles become equivalent in the thermodynamic limit (which in most cases is reached already in not too large systems, as shown by the simulations with few degrees of freedom).

The two viewpoints differ microscopically in term of the representation of reversibility, but provide the same statistical mechanical picture.

More precisely some among the equivalence properties that we consider are:

(1) If $\mu_{\overline{\mathbf{E}}}(\boldsymbol{\alpha})=\boldsymbol{\nu}$ then $\mu_{\boldsymbol{\nu}}(\mathbf{E})=\overline{\mathbf{E}}$ : the equivalence is reflexive.

(2) If $g(x)$ is a smooth observable in a large class (for instance in the case of the NS equations if its Fourier transform can be expressed in terms of few low Fourier modes), the statistics of $\gamma \stackrel{\text { def }}{=} \frac{1}{T} \int_{0}^{T} g\left(S_{t} x\right) d t$ is the same in the two systems for $T$ large 
(3) The fluctuation relation, [19], holds for the dissipation function

$$
\sigma(x) \stackrel{\text { def }}{=} \sum_{j} \partial_{j}\left[\left(\partial_{j} \mathbf{E}(x)\right) \cdot M^{-1}(x) \mathbf{\Phi}(x)\right]
$$

which in the example of Eq.(1.8) and for $\mathbf{g}_{\mathbf{k}} \neq 0$ only for few small $\mathbf{k}$ (i.e. for large scale forcing) is

$$
\begin{aligned}
\sigma(\mathbf{u})= & 2\left(\sum_{\mathbf{k}} \mathbf{k}^{2}\right) \alpha(\mathbf{u})+\frac{\sum_{\mathbf{k}} \mathbf{k}^{4} \mathbf{g}_{\mathbf{k}} \cdot \mathbf{u}_{-\mathbf{k}}}{\sum_{\mathbf{k}} \mathbf{k}^{4}\left|\mathbf{u}_{\mathbf{k}}\right|^{2}} \\
& -2 \frac{\left(\sum_{\mathbf{k}} \mathbf{k}^{2} \mathbf{g}_{\mathbf{k}} \cdot \mathbf{u}_{-\mathbf{k}}\right)\left(\sum_{\mathbf{k}} \mathbf{k}^{6}\left|\mathbf{u}_{\mathbf{k}}\right|^{2}\right)}{\left(\sum_{\mathbf{k}} \mathbf{k}^{4}\left|\mathbf{u}_{\mathbf{k}}\right|^{2}\right)^{2}} \simeq 2\left(\sum_{\mathbf{k}} \mathbf{k}^{2}\right) \alpha(\mathbf{u})
\end{aligned}
$$

where the sum over $\mathbf{k}$ is over the modes below the ultraviolet cut-off used 1

This means that if $\bar{\sigma}$ denotes the average value of the dissipation function in the stationary states $\mu_{\overline{\mathbf{E}}}^{i}$ and if $p=\frac{1}{\tau} \int_{0}^{\tau} \frac{\sigma\left(S_{t} x\right)}{\bar{\sigma}}$ then the probability $P_{\tau}(p) d p$ of the event $p \in d p$ in the distribution $\mu_{\overline{\mathbf{E}}}$ is multifractal and

$$
\frac{1}{\tau} \log \frac{P_{\tau}(p)}{P_{\tau}(-p)}=\bar{\sigma} p+O\left(\tau^{-1}\right)
$$

A more mathematical statement is that the rate function $\gamma(p)$ describing the multifractal distribution ${ }^{2}$ of the random variable $p$ with respect to $\mu_{\overline{\mathbf{E}}}$ has the symmetry property $\gamma(-p)=\gamma(p)-\bar{\sigma} p$ for $|p|<p^{*}$ where $p^{*}$ is a quantity depending on the system, however $p^{*} \geq 1$, for details see 9 .

(4) The Lyapunov spectra of the two distributions coincide.

\subsection{Our Model}

The goal of this paper is to substantiate the conjecture of equivalence between an irreversible dissipative-balanced model and the corresponding reversible model, constructed as detailed above. We consider the so-called Lorenz '96 model, proposed by E. Lorenz in a series of papers [27, 26]:

$$
\dot{x}_{j}=x_{j-1}\left(x_{j+1}-x_{j-2}\right)+F-\nu x_{j}, \quad j=0, \ldots, N-1
$$

\footnotetext{
${ }^{1}$ In 3 dimensions the natural cut-off would be the Kolmogorov scale; in 2 dimensions the cascade is inverse and the interpretation is more subtle, see [11].

${ }^{2}$ This is a function $\gamma(p)$ such that the probability the $p \in \Delta$ is asymtpotic as $\tau \rightarrow \infty$ to const $e^{\tau \max _{p \in \Delta} \gamma(p)}$ : in Anosov systems it is analytic, 41, 18, in $p$ for $|p|<p^{*}$ for some $p^{*}$; in time reversal symmetric Anosov systems $p^{*} \geq 1$.
} 
and periodic conditions $x_{k}=x_{N+k}, \forall k$. The r.h.s. of the equations has the typical structure of a sum of an energy conserving and time reversal invariant part plus a forcing part plus a dissipation violating time reversal (which in this case is the map $(I x)_{j} \stackrel{\text { def }}{=}-x_{j}$ ).

The variables $x_{j}$ of the model 1.14 have been originally interpreted as the value of a generic meteorological quantity measured around a latitudinal circle at a regular longitudinal interval.

The system shows chaotic motions, with a substantial fraction of positive Lyapunov exponents, already at moderate forcing i.e. at values of $R=\frac{F}{\nu^{2}} \geq$ 8: via the scaling $x_{j}(t)=\nu X_{j}(\nu t)$ the equation acquires the dimensionless form

$$
\dot{X}_{j}=X_{j-1}\left(X_{j+1}-X_{j-2}\right)+R-X_{j}, \quad j=0, \ldots, N-1
$$

The model is has become standard test-bed for predictability studies [32, for devising advanced data assimilation techniques [44, 43] and for constructing new parametrizations [45], so that today it has become the toy-model for studies of the nonlinear properties of geophysical flows.

Moreover, such a model has been extensively studied for constructing response operators to perturbations [1] and for testing accurately Ruelle's response theory [31, 30]. The applicability, up to an extremely high degree of precision of the Ruelle theory, described in [31] for $R=8$ supports the idea that the system features Axiom A-like properties i.e. - is sufficiently chaotic - already at moderate forcings.

Assuming the chaotic hypothesis, [20, 17], the system will reach a stationary state $\mu_{R}^{i}$ and $E=\frac{1}{2} \sum_{j=1}^{N} X_{j}^{2}$ will be a fluctuating variable with average value $\bar{E}_{R}^{i}=\int E(x) d \mu(x)_{R}^{i}$. We will consider as relevant macroscopic observable the momentum of the system, defined as $M=\sum_{j=1}^{N} X_{j}$, which will fluctuate around its average value $\bar{M}_{R}^{i}=\int M(x) d \mu(x)_{R}^{i}$.

The rescaled model Eq. $([1.15)$ ) fits into the general dissipation balanced form given in Eq. 1.1 when taking $k=1, E(X)=\frac{1}{2} \sum_{j} X_{j}^{2}, F_{j}=R, \nu=1$.

We will test the four equivalence properties described in the previous section on the irreversible and reversible versions of the Lorenz '96 model for a large range of value of $R$ in the turbulent regime. We want to emphasize that all the computations presented in this paper have ben performed on a regular laptop computer equipped with MATLABß V. 7.9 using a net total of about 10 days of CPU time.

The paper is organized as follows. In Sec 2 we describe some basic properties of the irreversible Lorenz '96 model, in particular confirming simple scaling laws describing the statistical properties of $M, E$, and showing new 
scaling laws for the spectrum of Lyapunov exponents. In Section 3 we describe the properties of the reversible Lorenz '96 model, showing that the conjectured equivalence holds to a very high precision, and in particular we discuss the validity of the fluctuation relation. In Sec. 4 we draw our conclusions. Finally, in the two Appendices we provide the scripts used to perform the computations presented in this paper.

\section{Properties of the Irreversible Lorenz '96 Model}

A good starting point in the investigation of the model given in Eq.1.15) is to consider the time evolution of $E$ :

$$
\dot{E}=-2 E+R M
$$

which implies that at steady state $\bar{E}_{R}^{i}=\frac{1}{2} R \bar{M}_{R}^{i}$.

The properties of Eq. 1.15) have been studied in detail in a number of papers. We consider the case where $N$ is sufficiently large (e.g. $N \geq 20$ ), so that it is possible to define stable (with respect to $N$ ) intensive properties (see e.g. [23]). Unless otherwise stated, all presented results refer to the case $N=32$. It is found that for $R<8 / 9$ the system features as unique attractor the fixed point $X_{j}=R, \forall j$, while for larger values of $R(<\sim 5)$ periodic motions due to nonlinearly equilibrated waves are observed. For $R \geq 5$, the system is in a turbulent regime, featuring multiple positive Lyapunov exponents. We will focus on the turbulent regime, and consider in the rest of our analysis $R \geq 8$. Our simulations have been run for $R=2^{p}$, $p=3, \ldots, 11$. The statistics have been collected on $10^{4}$ time units after discarding an initial transient. The equations have been integrated using the MATLABß V. 7.9 routine ode45, which allows for imposing a given relative and absolute precision in the integration through adaptive steps. A template for the MATLAB $\cap$ scripts used for investigating the statistical properties of $E$ and $M$ for the Lorenz '96 system is given in Appendix A. With small modifications, the scripts can be used also in Gnu-Octave (3.6.4-3). We have selected a relative and absolute precision of $10^{-8}$, which ensures extremely high precision in the simulation. As already observed by Lorenz [26], in such a regime the long term average of the energy $\bar{E}_{R}^{i}$ obeys accurately a scaling law as follows:

$$
\frac{\bar{E}_{R}^{i}}{N} \sim c_{E} R^{4 / 3} \quad c_{E}=0.59 \pm 0.01
$$


Considering Eq. (2.1), we derive:

$$
\frac{\bar{M}_{R}^{i}}{N} \sim 2 c_{E} R^{1 / 3}
$$

We have detected numerically several other scaling laws of considerable interest when studying higher order moments of the distribution of $E$ and $M$. The first significant result refers to the standard deviation of the energy $\operatorname{std}(E)_{R}^{i}=\left({\overline{E^{2}}}_{R}^{i}-\left(\bar{E}_{R}^{i}\right)^{2}\right)^{1 / 2}:$

$$
\frac{\operatorname{std}(E)_{R}^{i}}{N}=\tilde{c}_{E} R^{4 / 3}, \quad \tilde{c}_{E} \sim 0.2 c_{E} .
$$

The distribution of $E$ is to a good approximation Gaussian, and for each value of $F$ its standard deviation is proportional to the mean value. The standard deviation of $M$ can be described for $R \geq 200$ as

$$
\frac{s t d(M)_{R}^{i}}{N}=\tilde{c}_{M} R^{2 / 3}, \quad \tilde{c}_{M} \sim 0.046 \pm 0.001 .
$$

so that in this regime $\operatorname{st}(M)_{R}^{i} / \bar{M}_{R}^{i} \sim 0.039 \pm 0.001 R^{1 / 3}$. Therefore, as $R$ grows, the relative size of the fluctuations of $M$ increases, so that it is more and more likely to observe negative fluctuations of $M$. As soon as $R \geq 2000$, the standard deviation of $M$ is larger than half of its mean value, and larger than the mean value itself when $R \geq 20000$. This will become relevant when studying in the next section the fluctuation relation for the reversible model. As a last diagnostic property of the system, we note that the decorrelation time of $M$, i.e. the time needed for the value of its autocorrelation function to reduce by a factor $e$, obeys the following scaling relation:

$$
t_{d e c, M} \sim c_{t, M} R^{-2 / 3} \quad c_{t, M}=1.28 \pm 0.01
$$

Quite naturally, the stronger the forcing, the more intense the turbulence, the faster the loss of memory of the system. In order to provide a synthetic picture of the dynamical properties of the system, we next analyze its spectrum of Lyapunov exponents [8], obtained using the classical Benettin et al. [2] algorithm. A template for the MATLABß scripts used for studying the Lyapunov exponents of the Lorenz '96 system is given in Appendix B. Also in this case, with small modifications, the scripts can be used also in Gnu-Octave (3.6.4-3). In Fig. 1, we present the spectrum of Lyapunov exponents for $R=256$ and $R=2048$ and plot also the value of $\pi(j)=\left(\lambda_{j}+\lambda_{N+1-j}\right) / 2$. The confidence intervals shown in Fig. 1 indicate 


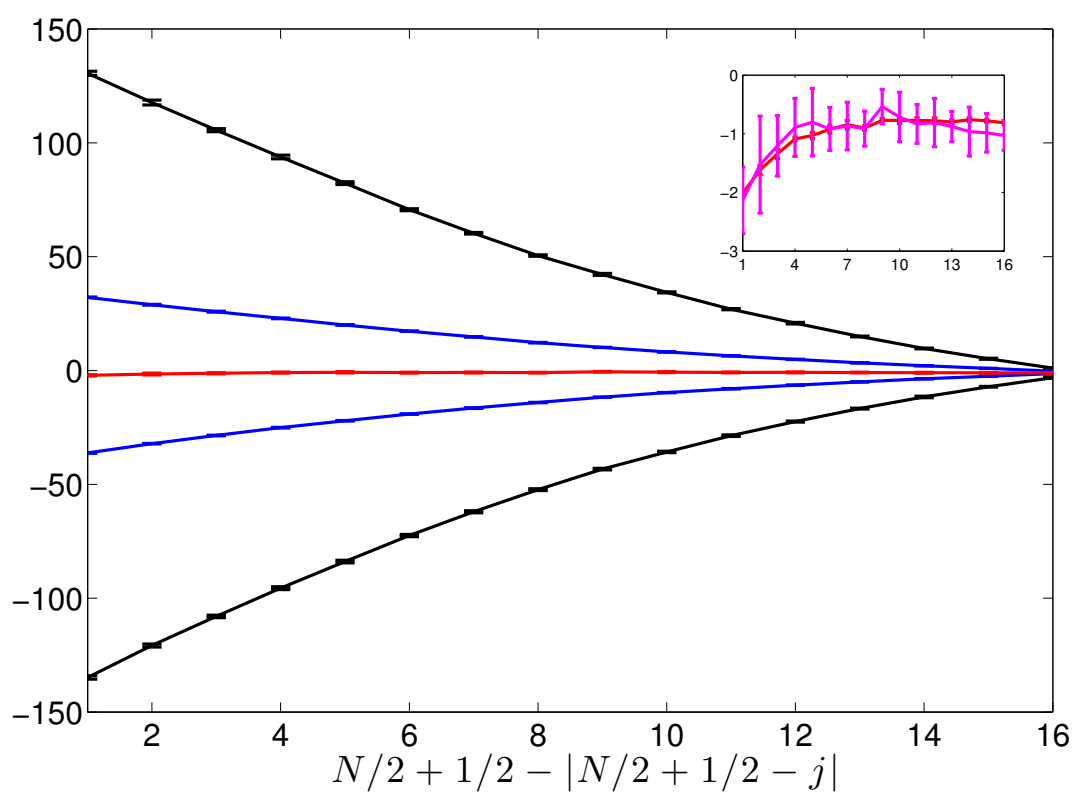

Figure 1: Black line: Lyapunov exponents for $R=2048$ arranged pairwise. Magenta line: corresponding value of $\pi(j)=\left(\lambda_{j}+\lambda_{N-j+1}\right) / 2$. Blue line: Lyapunov exponents for $R=256$ arranged pairwise. Red line: corresponding value of $\pi(j)=\left(\lambda_{j}+\lambda_{N-j+1}\right) / 2$. Irreversible model.

the $3 \sigma$-uncertainty range around the expectation value. For each $\lambda_{j}$ and $\pi(j), \sigma$ is computed as standard deviation of $n=10$ estimates of obtained running the model at steady state for $1000 t_{d e c, M}$ time units starting from different initial conditions, divided by the square root of $n$. The expectation value is computed, instead, as average of the $n$ estimates. As expected, the Lyapunov exponents increase with $R$, and, remarkably, each couple of Lyapunov exponents sums up to a value smaller in absolute value than each of them, and smaller than zero. Let's investigate this further.

It is known that the Lyapunov exponents $\lambda_{j}$ of a system consisting of a Hamiltonian evolution plus a constant forcing plus a linear dissipation with constant coefficient $\nu$ have the property $\lambda_{j}=-\lambda_{N+1-j}$ ), [6, 5]. The time translations of the inviscid and unforced dynamics in the r.h.s. Eq. (1.15) are reversible (hence have at least a common property with the usual Hamiltonian evolutions), and the friction coefficient and the forcing are independent of $X$, it is tempting to interpret Fig. 1 using such framework. 
Some remarks need to be made. In [3] it is explained that the inviscid and unforced dynamics is not Hamiltonian. As a result, the Jacobian matrices along the trajectories (which live on the spherical shell with energy set by the initial conditions) are not simplectic. Nonetheless, for such dynamics, we discover that the infinite time (but not the local) Lyapunov exponents obey a Hamiltonian-like pairing rule for any given value of the initial energy, so that $\lambda_{j}=-\lambda_{N+1-j}$ (not shown).

Results are presented in Fig. 2, where we plot $\pi(j)$ for $R=2^{2 q+1}, q=$ $1, \ldots, 5$, excluding the other considered values of $R$ for reasons of readability. We observe an emerging rule of pairing to a quite well defined $R$-independent curve over a large range of values of $R, 8 \leq R \leq 20483^{3}$ The figure shows that for all $j$ 's the width of the confidence interval increases with $R$, basically because $\pi(j)$ is constructed as algebraic sum of two quantities of opposite sign, whose average values and fluctuations increase in magnitude with $R$.

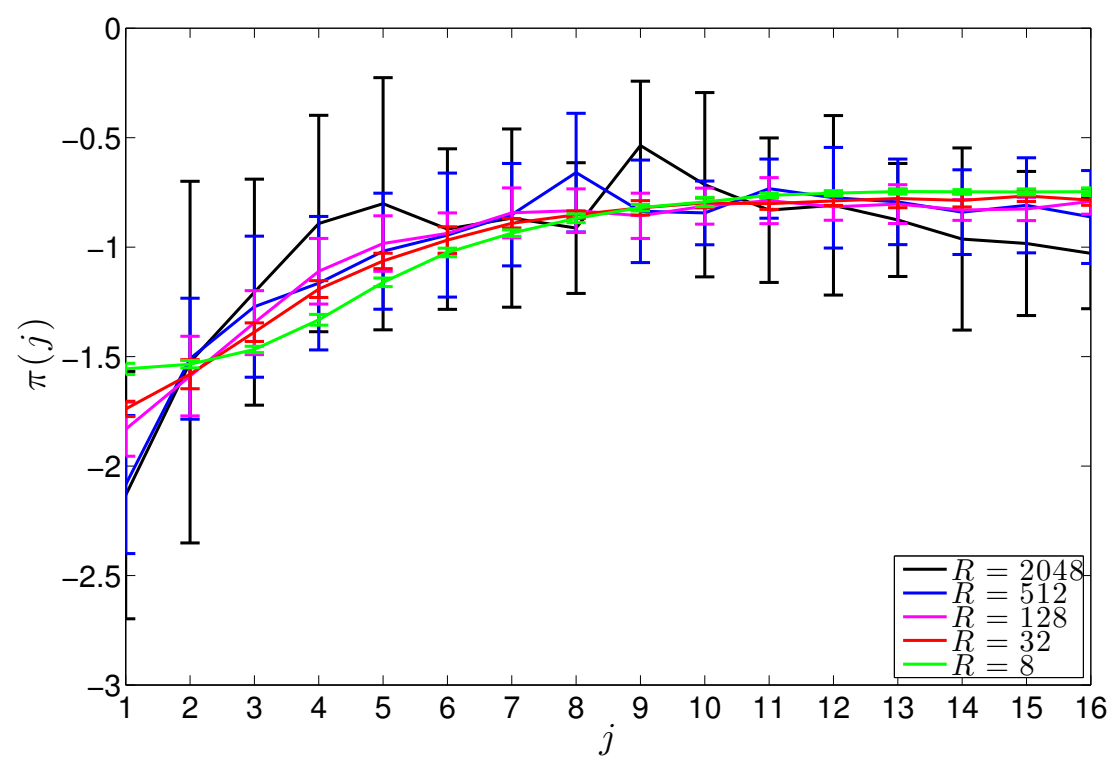

Figure 2: Pairing rule $\pi(j)=\left(\lambda_{j}+\lambda_{N-j+1}\right) / 2$ for selected values of $R$. Irreversible model.

While the fact that observables such as $\bar{E}_{R} / N$ and $\bar{M}_{R} / N$ weakly depend

\footnotetext{
${ }^{3}$ Also featuring modest deviations , compared to the size of the strongly $R$-dependent Lyapunov exponents, from what we would have obtained (a constant -1 value) had the inviscid, unforced dynamics been Hamiltonian.
} 
on $N$ is quite intuitive, we want to substantiate the fact that the spectrum of the Lyapunov exponents of the system does not depend asymptotically on the value of $N$, so that as $\lim N \rightarrow \infty$ one expects, see e.g. [24], to find a density of Lyapunov exponents. We report in Fig. 3 the spectrum of Lyapunov exponents obtained for $R=256$ and $N=256$, which is very similar to what reported in Fig. 1 for the corresponding value of $R$ once we rescale $j$ to $j / N+1$. While the confidence intervals do not overlap, we are confident that convergence is obtained with increasing values of $N$. In Fig. 3 ,

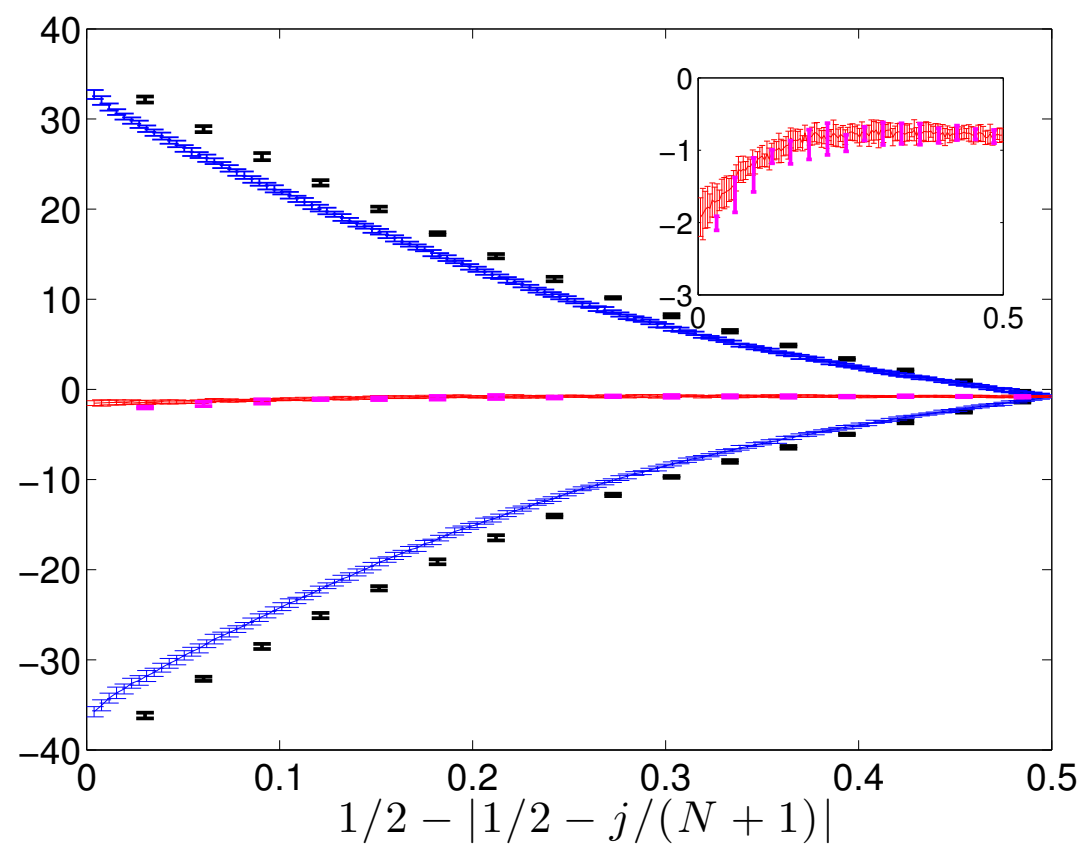

Figure 3: $R=256$ : Lyapunov exponents $\lambda_{j}$ for $N=256$ (blue line) and $N=32$ (black markers). Values of $\pi(j)=\left(\lambda_{j}+\lambda_{N-j+1}\right) / 2$ for $N=256$ (red line) and $N=32$ (red marker); a zoom is provided in the insert. Irreversible model.

we also report $\pi(j)$ for $R=256$ and $N=256$, which is in excellent agreement with what reported in Fig. 1, with overlapping confidence intervals. In the following, we will refer to the thermodynamic limit $N \rightarrow \infty$, where the discrete set of the Lyapunov exponents indices $j=1, \ldots, N$ is replaced by the continuous variable $x \in[0,1]$.

Accurate scaling laws can be found also for the Lyapunov exponents, 


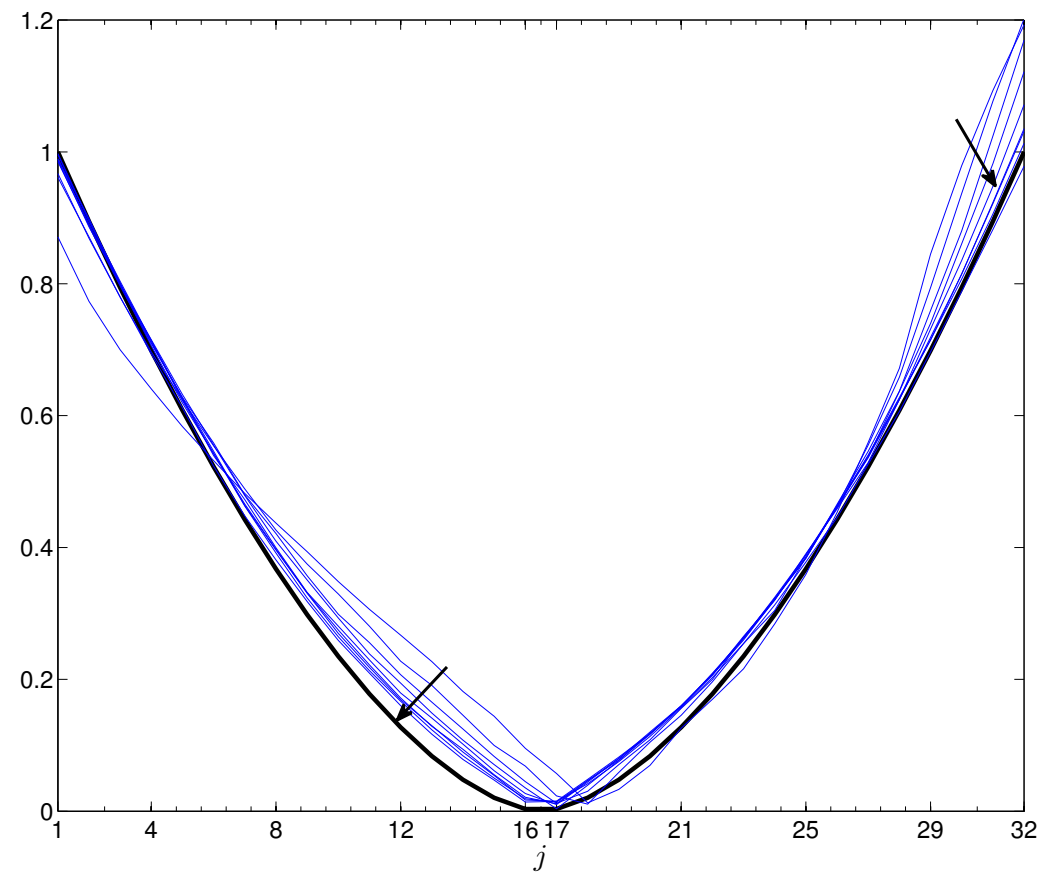

Figure 4: Blue lines: $\left|\lambda_{j}+1\right| /\left(c_{\lambda} R^{2 / 3}\right)$ for different $R$ (growing as indicated with the arrows from 8 to 2048 as powers of 2). Black line: $|2 j /(N+1)-1|^{5 / 3}$. Irreversible model. See Eq. 2.8

so that it is possible to provide an extremely simple expression for the full spectrum. We find stringent numerical evidence that

$$
|\lambda(x)+\pi(x)| \sim c_{\lambda}|2 x-1|^{5 / 3} R^{2 / 3}
$$

where $\pi(x)$ is depicted in Fig. 2 or Fig. 3 once we interpret the index $j$ as $(N+1) x$. The scaling relation given in Eq. 2.7$)$ can be safely approximated for large values of $R$ as:

$$
|\lambda(x)+1| \sim c_{\lambda}|2 x-1|^{5 / 3} R^{2 / 3}
$$

Remark: In this case the largest Lyapunov exponent scales independently of $N$ : a property that has been debated and is in agreement with [24].

In Fig. 4 we provide a graphical evidence of Eq.(2.8) ; we do not plot the confidence intervals of the rescaled $\lambda$ 's as their width is comparable to or 
smaller than the width of the lines. Note that our results seem to disagree with [23], where very different empirical scaling laws are reported and tested on a much more limited range of values of $R$ (up to about 30). While in the common range of $R$ the results agree with what reported in [23], we believe that the scaling laws given here are more general because they provide an excellent model for the obtained values of $\lambda$ 's in a much wider range of values of $R$. Moreover, it is clear that the expressions given in [23] cannot be used for studying the limit of $R$ going to infinity. The knowledge of $\lambda(x)$ allows one to compute several relevant properties of the invariant measure of the system. We first find the position $x_{0}$ of the vanishing Lyapunov exponent:

$$
\lambda\left(x_{0}\right)=0 \rightarrow x=x_{0}=\frac{1}{2}-\frac{R^{-2 / 3}}{2 c_{\lambda}^{3 / 5}}
$$

Obviously, if our system has $N$ degrees of freedom, we expect that $\lambda_{j}=0$ for $j=\left\lceil N x_{0}-0.5\right\rceil$. It is then straightforward to derive the expression metric entropy, estimated as the sum of the positive Lyapunov exponents [35]:

$$
\frac{\eta}{N}=\int_{0}^{x_{0}} d y \lambda(y)=\frac{3}{16} c_{\lambda} R^{2 / 3}-\frac{1}{2}+\frac{5}{16} R^{-2 / 5} c_{\lambda}^{-3 / 5},
$$

where the expression is valid in the limit of $N \rightarrow \infty$, and finally one can derive a closed expression for the Kaplan-Yorke dimension $d_{K Y}$ defined as:

$$
\frac{d_{K Y}}{N}=\tilde{y} \quad \text { with } \quad \int_{0}^{\tilde{y}} d y \lambda(y)=0
$$

In our case, since in our parametric range one can see that $\tilde{y} \geq 1 / 2$, we derive:

$$
\int_{0}^{1 / 2} d y\left(c_{\lambda}(1-2 y)^{5 / 3} R^{2 / 3}-1\right)+\int_{1 / 2}^{\tilde{y}} d y\left(-c_{\lambda}(2 y-1)^{5 / 3} R^{2 / 3}-1\right)=0
$$

We derive:

$$
\int_{\tilde{y}}^{1} c_{\lambda}(2 y-1)^{5 / 3} R^{2 / 3}=\tilde{y}
$$

solving the integral we obtain:

$$
\frac{3}{16} c_{\lambda} R^{2 / 3}\left(1-(2 \tilde{y}-1)^{8 / 3}\right)=\tilde{y}
$$

This is an implicit formula for the Kaplan-Yorke dimension. Let's explore the limit where $\tilde{y} \sim 1$. Assuming $1-\tilde{y} \ll 1$ we derive:

$$
c_{\lambda} R^{2 / 3}(1-\tilde{y})=\tilde{y}
$$


So that:

$$
\tilde{y}=1-\frac{1}{1+c_{\lambda} R^{2 / 3}}
$$

Therefore, if we have:

$$
N-d_{K Y}<1 \rightarrow \frac{N}{1+c_{\lambda} R^{2 / 3}}<1
$$

the attractor has a dimension virtually indistinguishable from that of the full phase space. If $R$ is very large, the system's attractor occupies a phase space volume close to the one ergodically visited in the inviscid, not forced system or, equivalently, one could say the system is weakly damped. Note that the last inequality can be read as $N<\left|\lambda_{N}\right|$, which agrees with what would be obtained without resorting to the continuous variable $x$, except for a term of order 1 ( $N-1$ instead of $N$ in the l.h.s.).

\section{Equivalence between the Reversible and Irre- versible Versions of the Lorenz'96 Model}

Following the indications given in Eq.(1.2)-(1.4), we construct the candidate equivalent time-reversible model as follows:

$$
\begin{aligned}
& \dot{X}_{j}=X_{j-1}\left(X_{j+1}-X_{j-2}\right)+R-\alpha(X) X_{j}, \quad j=0, \ldots, N-1 \\
& \alpha(X)=R \frac{\sum_{j} X_{j}}{\sum_{j} X_{j}^{2}}=\frac{R M}{2 E},
\end{aligned}
$$

plus the imposed periodic conditions $X_{k}=X_{N+k} \forall k$. The model conserves exactly the energy $E=\sum_{j=1}^{N} X_{j}^{2} / 2$. Following the procedure described in Sec. 1, we initiate each simulation with a given $R$ using initial conditions $X_{j}(0) j=1, \ldots, N$, such that $E(0)=\bar{E}_{R}^{i}$, which makes sure that the energy of the flow realized by the reversible model has constant energy equal to the average energy observed in the corresponding irreversible model forced with the same value of $R$.

Geometrically, the motion of the reversible model is constrained to the spherical surface defined by the initial conditions and will reach a stationary state defined by the measure $d \mu(x)_{R}^{r}$. In order to simulate accurately such a delicately constrained dynamics it is extremely important to use an integrator of ordinary differential equations able to use adaptive step and allowing for pre-defined relative and absolute error in the integration. Using 
the MATLABßV. 7.9 routine ode45 proved crucial for obtaining accurate results, because we could keep the same standard of accuracy in the irreversible and reversible runs. See Appendix A. As a result of numerical errors, a very small amount of energy is lost with time in the runs of the reversible model. Such spurious loss increases with $R$ but we have managed to limit it in relative terms to less than $10^{-6}$ with respect to the initial value between the beginning and the end of the simulations.

Clearly, for a given value of $R$, the support of the invariant measures $d \mu_{R}^{i}$ and $d \mu_{R}^{r}$ of the irreversible model (see Eq.11.15) and of the corresponding reversible model (see Eq.(3.1)), respectively, are substantially different, because $E$ in the irreversible model fluctuates by $20-40 \%$ (see Eq.(2.4)). Despite the fact that the two attractors are clearly not coincident, the conjectured equivalence holds to a very high degree of approximation.

The first observation is that for all considered values of $R$ the expectation value of $\alpha(X)$ is 1 within 1\%, in agreement with property (1) in Sec. 1.1 . As for point (2), we consider as natural test observable $g(x)$ the quantity $M$. Also in this case, we observe that $\bar{M}_{R}^{i}$ and $\bar{M}_{R}^{r}$ agree within $1 \%$, and the applies same when comparing ${\overline{M^{2}}}_{R}^{i}$ and $\bar{M}^{2}{ }_{R}^{r}$. In Fig. 5 we show the probability distribution of $M$ for $R=512$ in order to give a feeling of the strong similarity between the probability distribution functions (pdfs) of $M$ for two long $R=256$ integrations performed using the reversible and irreversible model. Another finding is that, for the same $R$, the decorrelation time of $M$ in the reversible and irreversible models agree accurately, so that also for the reversible model we can write $t_{d e c, M}=c_{t, M} R^{-2 / 3}$.

We now would like to address property $(\mathbf{3})$ in Sec. 1.1. The goal is to investigate the fluctuations at different time scales of the quantity $\sigma(X)=$ $(N-1) \alpha(x)$. For this purpose, at a given value of $R$, we define:

$$
p_{\tau, R}^{x}=\frac{1}{\tau} \int_{0}^{\tau} \frac{\sigma\left(S_{t} x\right)}{\bar{\sigma}_{R}}
$$

as the $\tau$-averaged value of the contraction of the phase space evaluated starting from point $x$ initially chosen at random uniformly on the energy surface and, therefore, with statistical properties that with probability 1 coincide with those of a typical point on the attractor, described by the invariant $\mathrm{SRB}$ measure of the system defined by the parameter $R$, and consider that $\bar{\sigma}_{R} \sim N-1$ within $1 \%, \forall R$. Let's define $P_{\tau}^{R}(p) d p$ the probability (constructed according to the invariant measure of the system) of observing 


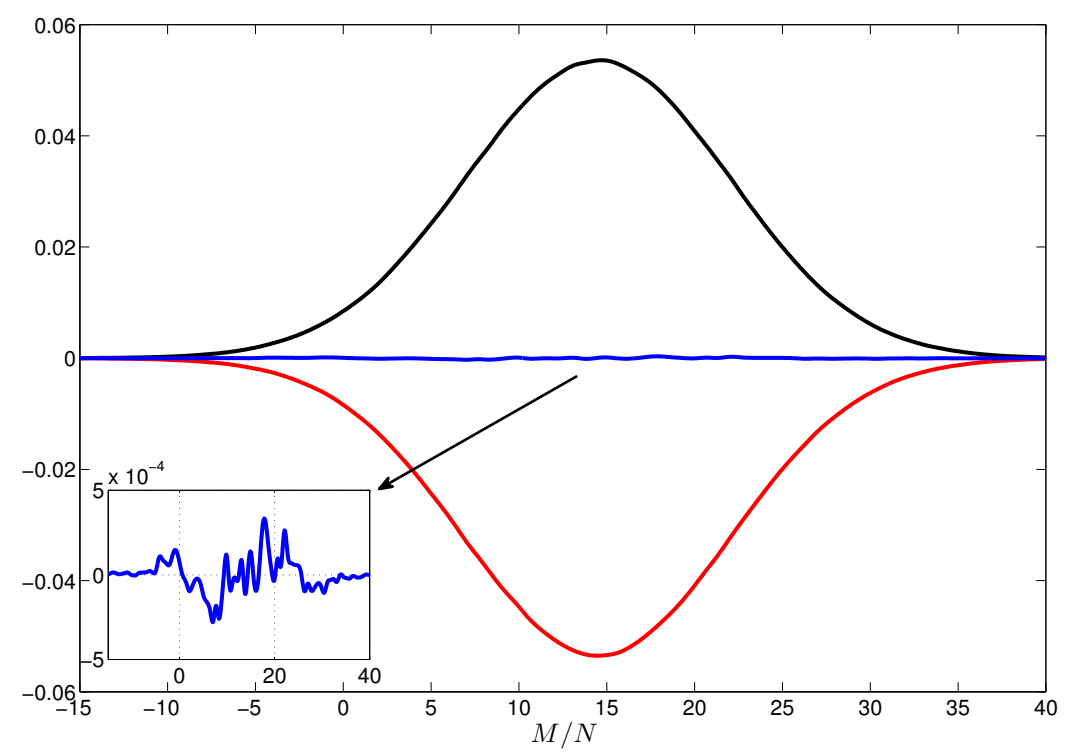

Figure 5: Black line: pdf for $M / N$ in the reversible model, $R=2048$. Blue line: opposite of the pdf for $M / N$ in the irreversible mode, for the same value $R=2048$. Red line: sum of the black and blue line. Note the different vertical scale in the insert.

$p_{\tau, R}^{x} \in[p, p+d p]$. We want to test whether the FR holds, namely, whether:

$$
\frac{1}{\tau} \log \left[\frac{P_{\tau}^{R}(p)}{P_{\tau}^{R}(-p)}\right]=\bar{\sigma}_{R} p+O\left(\tau^{-1}\right),
$$

and we want to study also which is the rate of convergence of the FR to its $\tau \rightarrow \infty$ limit, in order to substantiate the extent to which the FR can be verified, and be useful, when finite times (in the macroscopic time scales of the system) are considered.

We construct from the original time series of $\sigma(t)$ given as output of the reversible model the corresponding $\tau$-coarse grained time series. We then construct the $P_{\tau}$ probability distributions by either using non-parametric estimators such as histograms (tested for numerical stability) or, instead, Gaussian kernel smoothers [4].

The results presented here are weakly dependent on the statistical model used for estimating probability density, but using histograms it is easier to construct confidence intervals for the left hand side of Eq. 3.3. Therefore, 
we present findings obtained with histograms. We have that, since $\sigma=\frac{R M}{2 E}$, $\sigma / \bar{\sigma}_{R}$ and $M / \bar{M}_{R}^{r}$ are identical, so that, $t_{d e c, \sigma}^{R}=t_{d e c, M}^{R}$, where we have added explicit reference to $R$. From what discussed in Sec. 2, it is more likely to find negative fluctuations for the $\tau$-time averaged values of $\sigma$ when high values of $R$ are considered. We present results relative to $R=512$ and $R=2048$ in Figs. 667, respectively. We plot:

$$
\frac{1}{\tau \bar{\sigma}_{R}} \log \left[\frac{P_{\tau}^{R}(p)}{P_{\tau}^{R}(-p)}\right]
$$

against $p$. The $3 \sigma$ confidence intervals are computed by suitably performing bootstrap on the results obtained on steady state trajectories of length $10^{6} t_{d e c, \sigma}^{R}$. Three main observations can be made:

- For both values of $R$, the FR is obeyed to a good degree of accuracy for large - to be defined later - values of $\tau$;

- Before the asymptotic result is obtained, the quantity given in Eq. (3.4) is proportional to $p$ via a constant $c(\tau)$, which converges to 1 as $\tau$ becomes large;

- The larger $R$, the faster is the convergence to the FR with $\tau$.

The first observations basically provides a verification for point (3) of the conjecture proposed in Sec. 1.1. The second observation does not derive trivially from the FR given in Eq. $(3.3)$, because it implies that the $O\left(\tau^{-1}\right)$ correction is, in fact, proportional to $p$. Since $p$ is of order one, this result is anyway consistent with the FR. One can show that $O\left(\tau^{-1}\right) \propto p$ in the case of gaussian process, which, in fact, $P_{\tau}^{R}$ conforms to with a high degree of accuracy. It is possible to infer from numerical experiments a very simple expression for the corrective factor $c(\tau)$ :

$$
c(\tau)=1+\left(\frac{t_{d e c, \sigma}^{R}}{\tau}\right)^{4 / 3}=1+\left(\frac{c_{t, \sigma}}{\tau}\right)^{4 / 3} R^{-8 / 9},
$$

where $c_{t, \sigma}=c_{t, M}$, which explains the third observation above. Equation (3.5) is crucial for understanding the speed of convergence of the limit implied by the FR, and, conversely, for estimating how large $\tau$ must be in order to find good agreement with the FR. Interestingly, the time scale appearing in the formula is the decorrelation time of $\sigma$ itself, which is extremely easy to estimate and which we have observed to coincide with that of $M$. Roughly, as long as $\tau$ is larger than such time scale by a factor of say 10, we observe 


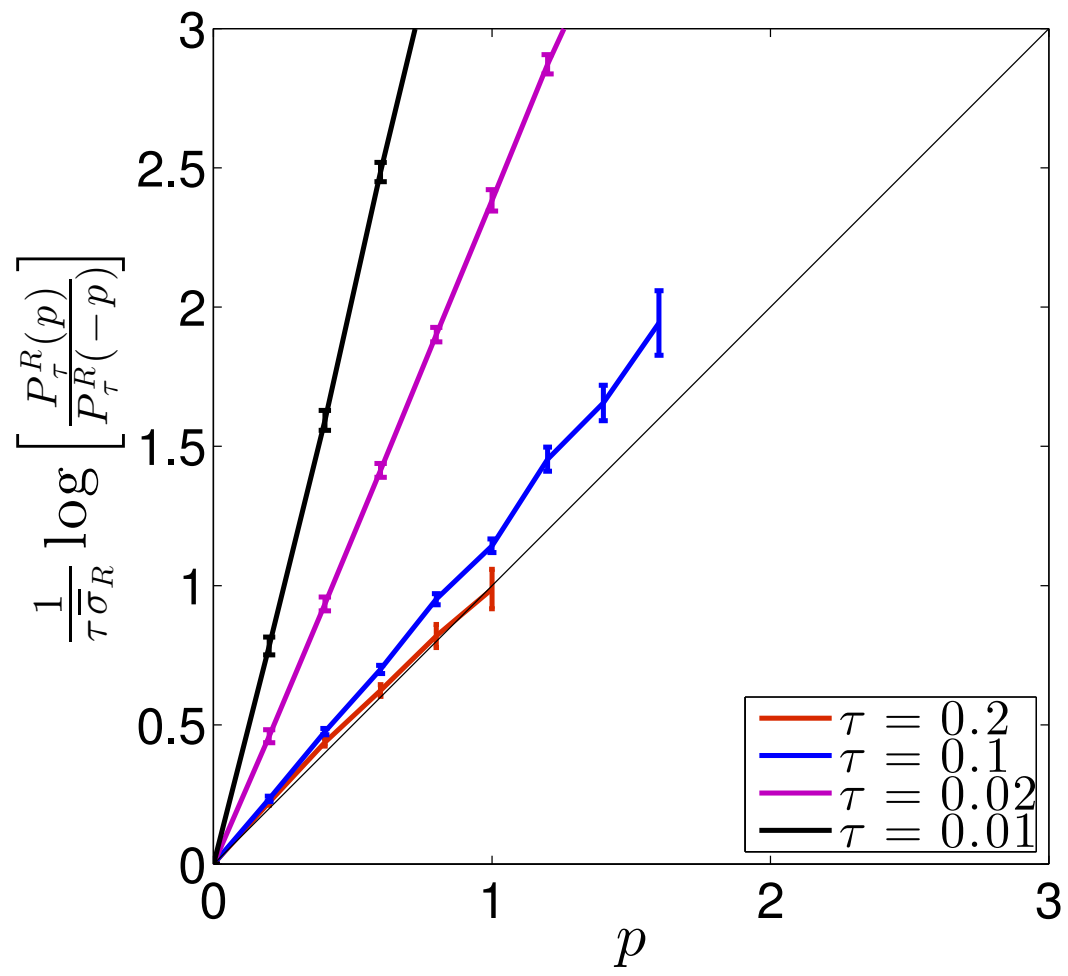

Figure 6: Fluctuation Relation for $R=512$, approaching slope 1 as $\tau$ increases. See Eq. 3.3 .

small deviations from the FR. Moreover, the corrective factor convergences to 1 with $R^{-8 / 9}$, indicating that as the forcing to the system becomes more intense, the FR relation can be observed for smaller and smaller averaging times $\tau$, going to 0 in the limit $R \rightarrow \infty$.

The FR is indeed signature of the a) time-reversible nature of the flow and b) of the compatibility with the chaotic hypothesis. Given the equivalence discussed above, it makes sense to check whether in the irreversible system one can find quantities obeying the FR.

We construct as obvious candidate is $\tilde{\sigma}_{R}=N R M /\left(2 \bar{E}_{R}^{i}\right)$. The first moments of this quantity, as discussed above referring to $M$, are almost identical to those of $\sigma_{R}$ in the reversible system. Nonetheless, when testing whether the FR holds even in this irreversible case, we find deviations with 


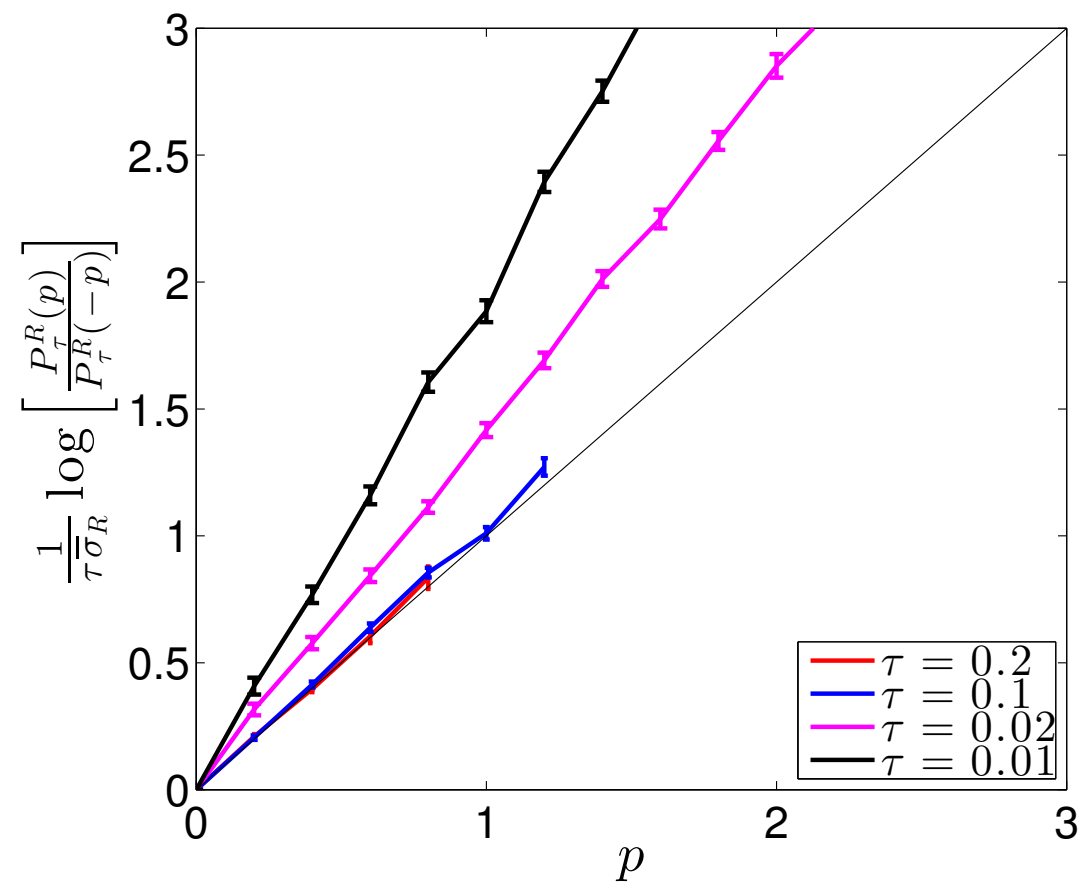

Figure 7: Fluctuation Relation for $R=2048$, with slope approaching 1 as $\tau$ increases beyond the decorrelation time. See Eq. (3.3). Note that the red line is hidden below the blue line.

respect to what obtained in the reversible case. In particular, we find that $c_{\tau}$ does not converge to 1 , with discrepancies of the order of $20 \%-30 \%$. The presence of such - not too large - departure from the FR might explain why some experimental and numerical attempts at verifying FR in irreversible systems, and so outside its realm of validity, have encountered some degree of success.

Following a suggestion first discussed in [13, 16, we have also attempted the verification of a local version of the FR, which amounts to studying the large fluctuations of some measure of the local contraction of the phase space. Therefore, we define ([13, Eq.(6.2)],[14, Eq.(9.2)])

$$
\sigma^{\beta}(X)=N R \frac{\sum_{j=1}^{N_{0}} X_{j}}{\sum_{j=1}^{N} X_{j}^{2}} .
$$

where the summation in the numerator is performed over $N_{0}$ nearby points, 
covering a portion $\beta=N_{0} / N$ of the total volume of the system (note that the system is invariant to discrete translation). In [13] it is proposed that (a) the expectation value of $\bar{\sigma}_{R}=\beta \bar{\sigma}_{R}$; and

(b) the quantity $p=\frac{\sigma^{\beta}}{\bar{\sigma}^{\beta}}$ obeys $\mathrm{FR}$, so that, defining as usual $p_{\tau, R}^{x}=$ $\frac{1}{\tau} \int_{0}^{\tau} \frac{\sigma^{\beta}\left(S_{t} x\right)}{\bar{\sigma}_{R}}$, and $P_{\tau}^{R}(p) d p$ the probability of observing $p_{\tau, R}^{x} \in[p, p+d p]$. we should have:

$$
\frac{1}{\tau} \log \left[\frac{P_{\tau}^{R}(p)}{P_{\tau}^{R}(-p)}\right]=\bar{\sigma}_{R}^{\beta} p+O\left(\tau^{-1}\right)=\beta \bar{\sigma}_{R} p+O\left(\tau^{-1}\right)
$$

We have tested these predictions for $R=2048$ by considering $N=32$ and $N_{0}=8$.

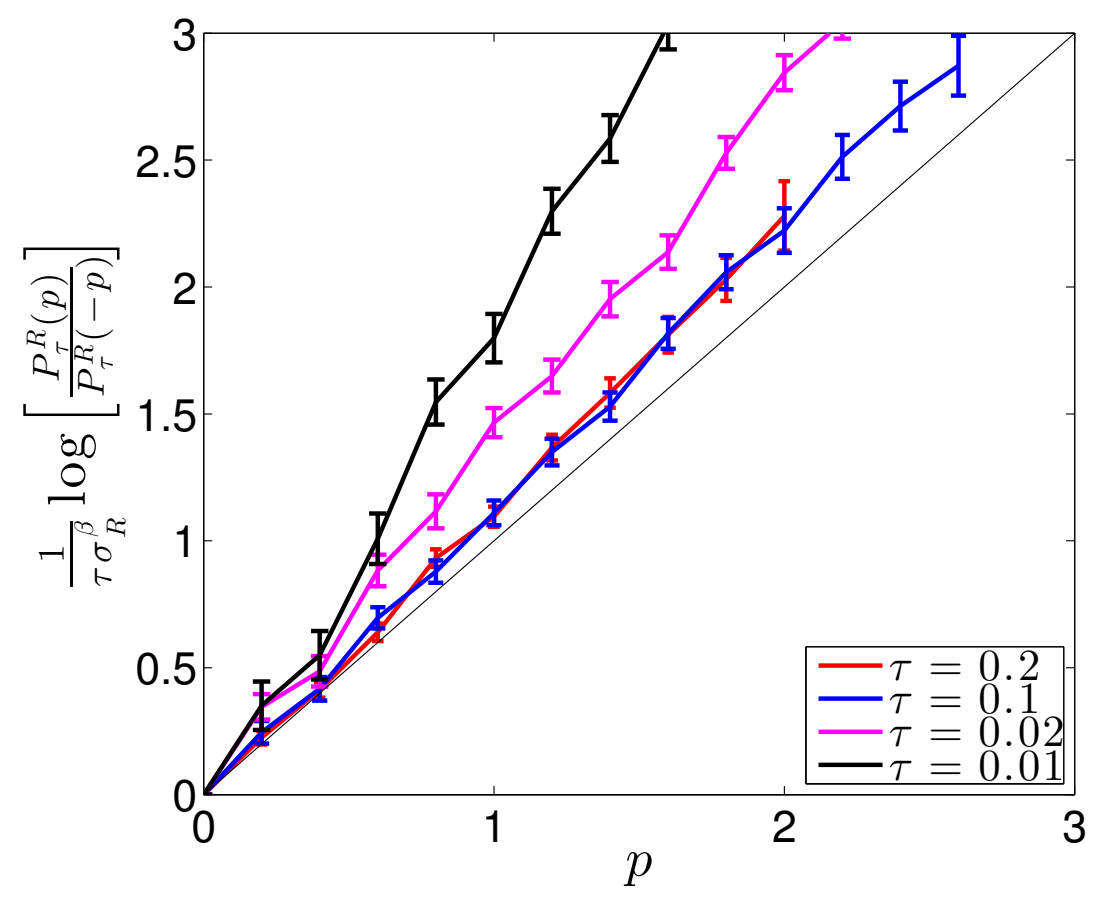

Figure 8: Local version of the Fluctuation Relation for $R=2048$. See Eq. 3.7. Note that the red line is hidden below the blue line.

The verification of property (a) is rather easy. Figure 8 shows that the local version of the FR proposed as property (b) is not perfectly obeyed (we observe a deviation of 5-10\%), possibly because of a finite size effect, 


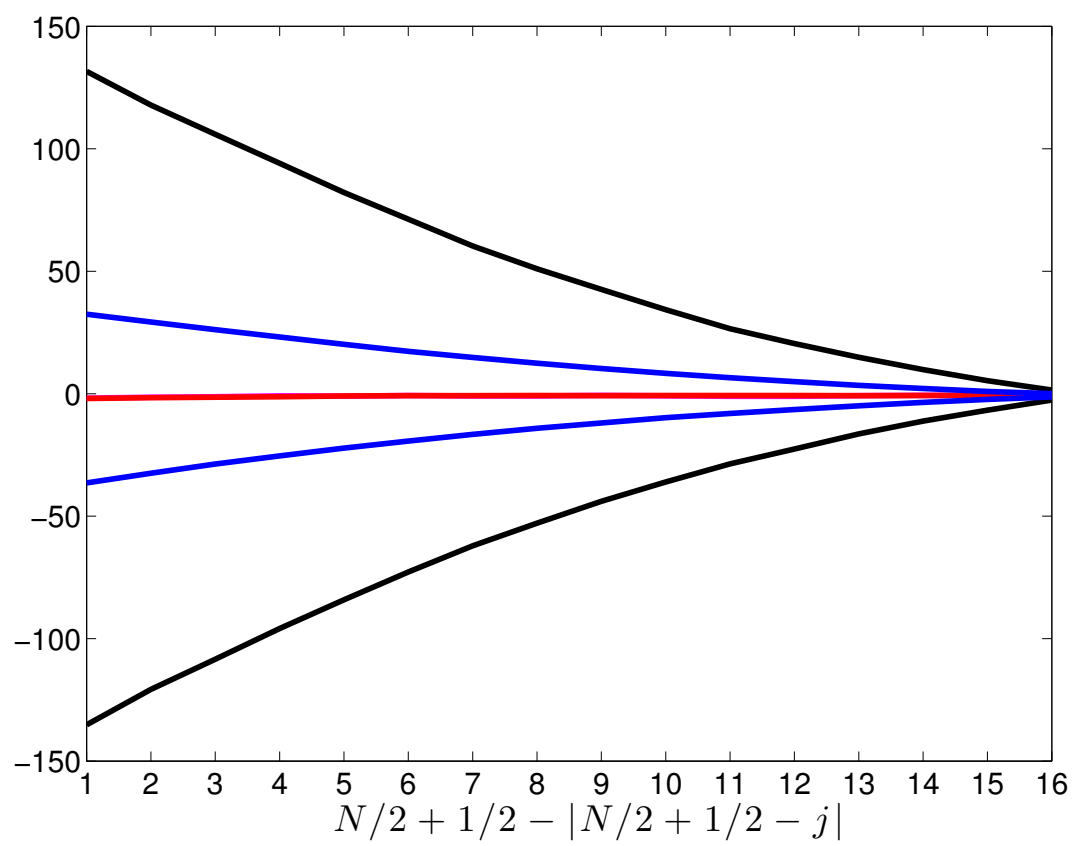

Figure 9: Black line: Lyapunov exponents for $R=2048$ arranged pairwise. Magenta line: corresponding value of $\left(\lambda_{j}+\lambda_{N-j+1}\right) / 2$. Blue line: Lyapunov exponents for $R=256$ arranged pairwise. Red line: corresponding value of $\left(\lambda_{j}+\lambda_{N-j+1}\right) / 2$. Reversible model (Compare with Fig. 1).

because in our experimental conditions we are far from the limits proposed in the conjecture above $\beta \rightarrow 0, N_{0}, N \rightarrow \infty$.

As for addressing point (4) in the list of properties discussed in Sec. 1.1. we compute the spectrum of the Lyapunov exponents of the reversible model given in Eq. 3.1. See Appendix B. It is important to note that, given the integral nature of the fluctuating viscosity $\alpha(X)$, the Jacobian matrix to be used in the Benettin et al. 2] algorithm is not anymore sparse, with resulting increased computational costs in terms of memory.

Our result provide strong evidence that the spectrum of Lyapunov exponents in the reversible and irreversible cases are indeed the same within statistical uncertainty (i.e. error bars overlap). In Fig. 9 we present the Lyapunov spectrum obtained for $R=256$ and $R=2048$ and $N=32$, which, by visual inspection, have a very close correspondence to what shown in Fig. 


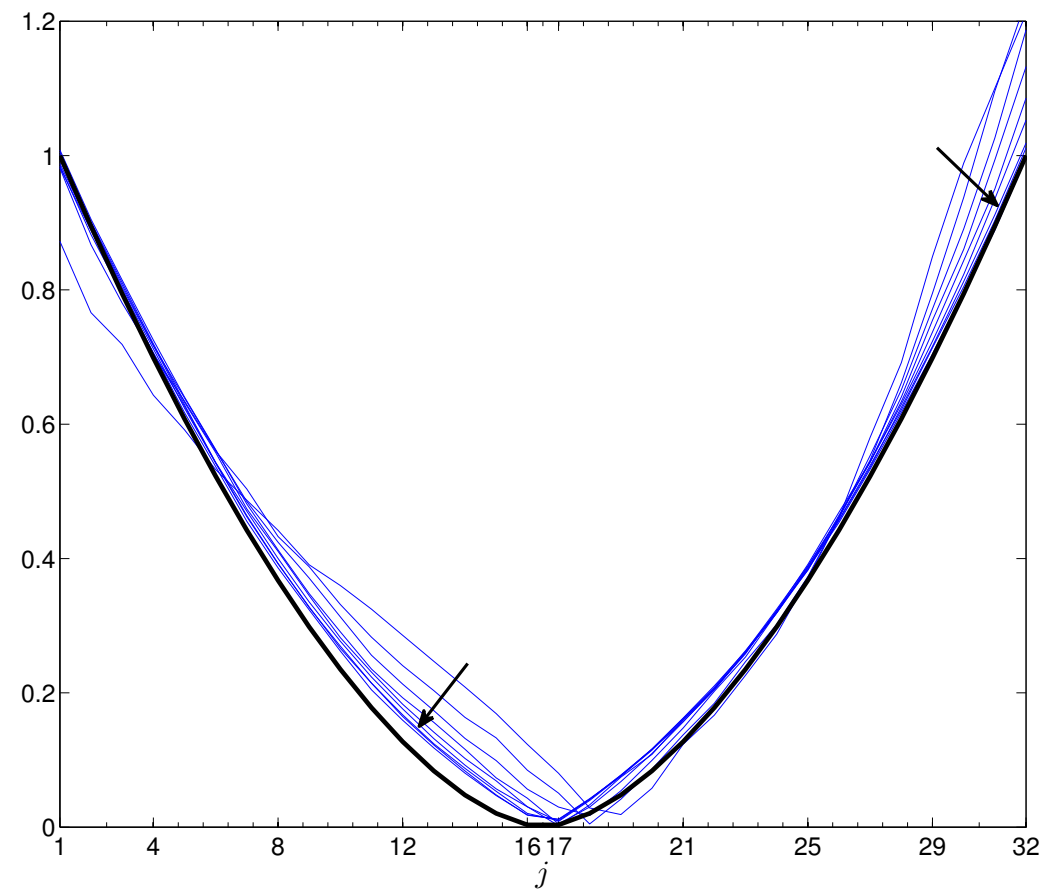

Figure 10: Blue lines: $\left|\lambda_{j}+1\right| /\left(c_{\lambda} F^{2 / 3}\right)$ for different $F$ (growing as indicated with the arrows from 8 to 2048 as powers of 2). Black line: $|2 j /(N+1)-1|^{5 / 3}$. Reversible Model. See Eq. 2.8 and compare with Fig. 3 .

1. Error bars are not reported in Fig. 9 to improve readability.

The quality of the agreement is clarified by the fact that also in the reversible case the approximate scaling law $\lambda(x)=c_{\lambda}(2 x-1)^{5 / 3} R^{2 / 3}$ applies; compare Fig. 10 with Fig. 3. The accuracy of the correspondence between the Lyapunov exponents of the irreversible and reversible case is further substantiated by the fact that we find virtually the same pairing rule $\pi(x)$ for all values of $\mathrm{R}$ (not shown) Since the scaling of the Lyapunov vectors agrees, and the pairing rule, which is obtained as the difference between two large numbers, is the same when comparing the irreversible and reversible case, we are drawn to the conclusion that also property (4) is indeed accurately obeyed. 


\section{Summary and Conclusions}

The possibility of providing macroscopically equivalent yet microscopically non identical descriptions of many-particle systems endows equilibrium statistical mechanics with a great flexibility in terms of theoretical calculations, and leads directly to a robust definition of thermodynamical properties. In this paper we have examined the problem of constructing equivalent ensembles for non-equilibrium statistical mechanical systems.

We have approached this problem by treating a case of irreversible dissipative balanced systems, a class of systems which includes the NS equations, characterized by a set of friction coefficients $\boldsymbol{\nu}$ and a corresponding set of quantities $\mathbf{E}$ which are conserved if forcing and dissipation are neglected, and fluctuate in time when forcing and dissipation are considered and the system reaches its invariant measure $\mu_{\boldsymbol{\nu}}^{i}$.

The corresponding reversible systems are constructed by changing the definition of constant friction into functions $\boldsymbol{\alpha}(x)$ in such a way that the quantities $\mathbf{E}$ are conserved (and set to the average values $\overline{\mathbf{E}}^{i}(\boldsymbol{\nu})$ of the functions $\mathbf{E}(x)$ in the distribution $\left.\mu_{\boldsymbol{\nu}}^{i}\right)$.

The attractor of such a system is the support of the invariant measure $\mu_{\overline{\mathbf{E}}}^{r}$. While the two invariant measures are indeed different, we can say that

$\mu_{\boldsymbol{\nu}}^{i}$ corresponds to $\mu_{\mathbf{E}}^{r}$ if $\mu_{\mathbf{E}}^{r}(\boldsymbol{\alpha})=\boldsymbol{\nu}$. We have proposed four criteria for defining the equivalence of the irreversible and reversible dynamics, which boils down to providing analogous information of the expectation values of smooth observables, to featuring an equivalent description of the instabilities of the system, and, in the spirit of the chaotic hypothesis, to featuring, in the case of the reversible system, a phase space contraction rate fluctuating in agreement with the FR.

While the long-term goal of this investigation is e.g. to study the equivalence between the customary irreversible representation of fluid-dynamics through the NS equations and its reversible version, in this paper we have considered a simple yet dynamically rich model introduced by E. Lorenz [27, 26]. Such a model describes advection, dissipation, forcing, in a ring of $N$ grid points, and has been the subject of extensive investigation in atmospheric sciences (see, e.g. [32, 45, 44, 43] ) and, more recently, in statistical physics (see, e.g. [1, 22, 31]). This model fits the paradigm of the irreversible dissipative balanced systems, so that, when forcing (described by the parameter $R$ ) and dissipation (a normalized viscosity $\nu$ is set to 1 ) are neglected, a quadratic quantity referred to as energy $E$ is conserved.

The Lorenz '96 system is strongly chaotic when the forcing parameter $R$ 
is larger than 8 and its properties are extensive with the number of nodes $N$. Our investigations have covered extensively the range $R=8$ up to $R=$ 2048, plus control runs performed at much higher value of $R$. Most of the simulations have been performed considering $N=32$, plus several additional runs performed with $N=256$ and $N=512$ to check how properties scale with $N$.

We have confirmed the existence of accurate scaling laws allowing to express the energy $E \sim R^{4 / 3}$ of the system and a suitably linear quantity referred to as momentum $M \sim R^{1 / 3}$. More interestingly, it is possible to find evidence of a simple scaling relation for the Lyapunov vectors $\lambda$ 's such that $|\lambda(x)+\pi(x)| \sim(2 x-1)^{5 / 3} R^{2 / 3}$, where $x$ refers to the normalized index $j /(N+1)$ in the continuous limit, and $\pi(x)$ is a $R$ independent pairing function, such that $\lambda(x)+\lambda(1-x)=2 \pi(x) \forall x \in[0,1]$ and (at least) for all the $R$ 's considered.

We have found numerical evidence for explicit expressions of the position of the zero Lyapunov exponent, of the metric entropy, and of the KaplanYorke dimension. The Kaplan-Yorke dimension saturates the dimension of the phase space as $R \rightarrow \infty$, as already observed by Lorenz [26], although the statistical properties, both in the reversible and in the irreversible models, are very different from those of the uniform distribution on the energy surface, i.e. from the equilibrium statistics in spite of the choice of the initial condition which are selected precisely with the latter distribution: a property familiar, from SRB theory of chaotic motions, in SRB distributions.

In the irreversible model $E$ fluctuates, while the contraction of the phase space is fixed. We can think this as analogous to the canonical ensemble, where the viscosity plays the role of temperature, while $E$ plays the role of energy.

Following the paradigm described above, we have constructed the (candidate) equivalent reversible system by introducing a fluctuating viscosity allowing for energy conservation and defining a time-reversible dynamics. Therefore, in this model the phase contraction fluctuates and energy is fixed, as opposed to the traditional irreversible model. We have verified for $\forall R$ excellent agreement between the reversible and irreversible model on (1) the average phase contraction rate; and (2) first moments of macroscopic observables (here we have taken $M$ as obvious candidate).

We have also verified (3) that the $\mathrm{FR}$ for the phase space contraction rate $\sigma_{R}$ is obeyed with good accuracy, and have highlighted that the convergence to the asymptotic result is controlled by the ratio between the averaging time $\tau$ over which the phase space contraction is evaluated and its $e$-folding time.

One finds that as long as $\tau$ is larger by a factor of say $5-10$, the FR 
is obeyed to a very good approximation. For any finite value of $\tau$, the correction to the FR result $\rightarrow 0 \sim R^{-8 / 9}$, so that for strong forcings the FR can be observed over very short averaging times. Interestingly, the finitetime correction to the asymptotic FR result appears as a multiplicative tending to 1 rather than additive factor going to 0 with $\tau \rightarrow \infty$, and can be related, in the Gaussian case, to the scaling of the variance of the distribution of $\tau$-averaged values of $\sigma_{R}$. We have also for the first time verified the validity of a local version of the FR proposed in [13] within a good degree of approximation.

Finally, we have also compared (4) the instability properties of the irreversible and reversible system by investigating the degree of agreement of the spectrum of Lyapunov exponents. We find that an extremely satisfying agreement, so that the same scaling laws and pairing rule are obeyed. When $R$ is very large, the system lives on an attractor whose closure is indistinguishable from the spherical shell defined by the initial value of the energy, thus resembling a micro-canonical ensemble.

We conclude by stressing that while the support of the two invariant measures $\mu_{R}^{i}$ and $\mu_{R}^{r}$ is indeed different, and the symmetries of the two corresponding dynamical systems also are different, numerical evidence supports, in the Lorenz96 system, that their statistical mechanical properties are virtually the same at all considered values of $R$ where turbulence is observed. For high $R$, we basically find an analog of the equivalence of canonical and microcanonical ensembles in chaotic systems.

In other words one may naively think that for high values of $R$ the constructed time-reversible system may, in fact, feature the same statistical mechanical properties of the system where forcing and dissipation are neglected altogether, if they live on the same energy shell. Note that value of $\bar{E}_{R}^{r}$ and $R$ are one-to-one related. This is, in fact, false. The first remark is that while the expectation value $\bar{M}_{R}^{r} \sim R^{1 / 3}$ in the time reversible system, in the corresponding inviscid and unforced system the expectation value of $M$ is, by symmetry, zero. Moreover, as discussed before, the Lyapunov exponents of the inviscid and unforced dynamics and of the time-reversible dynamics are indeed different (even if the difference is smaller and smaller in relative terms as larger and larger energies are considered). Note that the empirically defined pairing rule convergences for large values of $R$ to $\pi(x)$ which is indeed non-vanishing. Therefore, the SRB measure is distinct from the Gibbs measure also in the limit of $R \rightarrow \infty$, even though the attracting sets of the two measures become indistinguishable, with the sign of $R$ responsible for breaking the symmetry.

These results provide a strong motivation for considering the possibility 
of approaching the problem of modeling turbulent fluids using a radically different point of view, where, instead of setting to a constant the rate of contraction of the phase space, we keep constant some physical quantities of interest. This approach is expected to work more effectively when the nondimensional numbers describing the relative strength of forcing versus dissipation (e.g. the Reynolds number) is very large. One can think of applying this procedure only to specific regions of a fluid, as suggested by the local result in Fig. 8 ,

This seems promising in the case we have a fluid with strong anisotropy in one direction, like in the case of strong stratification, of special interest for geophysical applications. Moreover, these results clarify the relevance of the Chaotic Hypothesis for investigating turbulent fluid systems. In particular, under the assumption that the Chaotic Hypothesis holds, one can use the Ruelle response theory [36, 37] for studying the response of a system like the climate to forcings (see discussions in [28, 29] and some results in [31, 30, 34]).

As next steps in the line of investigation of this paper, one can envisage to approach the problem of studying the quasi-geostrophic turbulence in a realistic Earth-like setting, trying to go from a point of view where diffusion, Eckman friction, and viscosity are kept constant, to a point of view where the total energy and potential enstrophy at various levels are kept constant. 


\title{
A Script - Part 1: Integrating the Lorenz 96 irre- versible and reversible models
}

\author{
$\% \% \% \% \% \% \% \% \% \% \%$
}

$\% \% \%$ PART 1: Integrating the equations for the Loren6 96 $\% \% \%$ irreversible and reversible models

$\% \% \% \% \% \% \% \%$ Script by V. Lucarini (valerio.lucarini@uni-hamburg.de)

$\% \% \% \% \% \% \% \%$ Can be freely distributed according to GNU license.

$\% \% \% \% \% \% \%$ Companion Material to the paper

$\% \% \% \% \% \% \%$ Equivalence of Non-equilibrium Ensembles, $\% \% \% \% \% \% \%$ and Representation of Friction in Turbulent Flows: the $\% \% \% \% \% \%$ Lorenz 96 model, by G. Gallavotti and V. Lucarini, 2014 $\% \% \% \% \% \% \%$ values given below for the parameters $\% \% \% \% \% \% \%$ must be checked/optimized by the user

$\% \% \% \% \% \% \% \% \% \%$

$\%$ Lorenz 96 model - Irreversible version. Input parameters:

$\% \mathrm{~N}=$ number of modes ; $\mathrm{R}=\mathrm{R}$ parameter

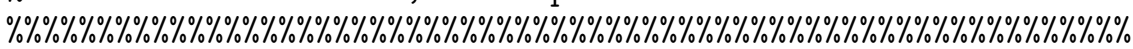

$\% \% \%$ save text below as lorenzabirr.m\%\% $\% \% \% \% \% \% \% \% \% \% \% \% \% \% \% \% \% \% \% \% \% \% \% \% \% \% \%$

function dy = lorenz96irr $(t, y, R, N)$

$\mathrm{dy}=\operatorname{zeros}(\mathrm{N}, 1)$;

$\mathrm{F}=$ ones $(\mathrm{N}, 1) * \mathrm{R}$;

$\operatorname{dy}(1)=(\mathrm{y}(2)-\mathrm{y}(\mathrm{N}-1)) * \mathrm{y}(\mathrm{N})-\mathrm{y}(1)+\mathrm{F}(1)$;

$\operatorname{dy}(2)=(y(3)-y(N)) * y(1)-y(2)+F(2)$;

$\operatorname{dy}(\mathrm{N})=(\mathrm{y}(1)-\mathrm{y}(\mathrm{N}-2)) * \mathrm{y}(\mathrm{N}-1)-\mathrm{y}(\mathrm{N})+\mathrm{F}(\mathrm{N})$;

for $j=3: N-1$

$\operatorname{dy}(j)=(y(j+1)-y(j-2)) * y(j-1)-y(j)+F(j)$;

end

$\% \% \% \% \% \% \% \% \% \% \% \% \% \% \% \% \% \% \% \% \% \% \% \% \% \% \% \% \% \% \% \% \% \% \% \% \% \% \% \% \% \% \% \% \% \% \% \% \% \% \% \% \% \% \% \% \% \% \% \% \% \% \% \%$

$\% \% \%$ save text above as lorenzabirr.m $\% \% \% \% \% \% \% \% \% \% \% \% \% \% \% \% \% \% \% \% \% \% \% \% \%$

\%\%\%\% \% \% \% \% \% \% \% \% \% \% \% \% \% \% \% \% \% \% \% \% \% \% \% \% \% \% \% \% \% \% \% \% \% \% \% \% \% \% \% \% \% \% \% \% \% \% \% \% \% \% \% \% \% \% \% \% \% \% \% \% \% \%

odeset

$\%$ Defines options for ode integrator (we use the standard

$\%$ ode45 function)

$\% \quad$ AbsTol: [ positive scalar or vector $\{1 \mathrm{e}-6\}$ ]

$\% \quad$ RelTol: [ positive scalar $\{1 \mathrm{e}-3\}$ ]

$\%$ NormControl: [ on | \{off\}]

$\% \quad$ NonNegative: [ vector of integers ] 


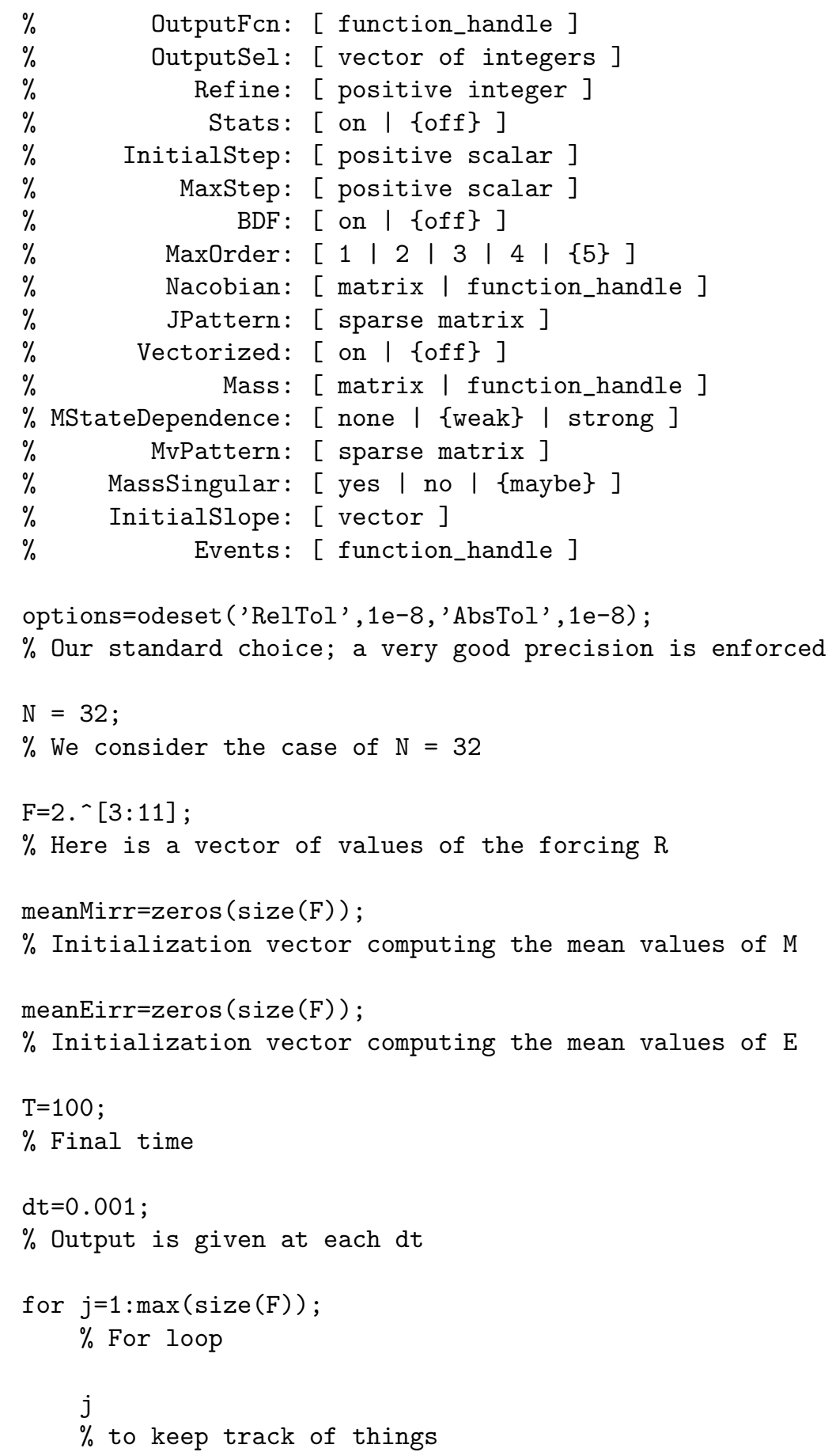




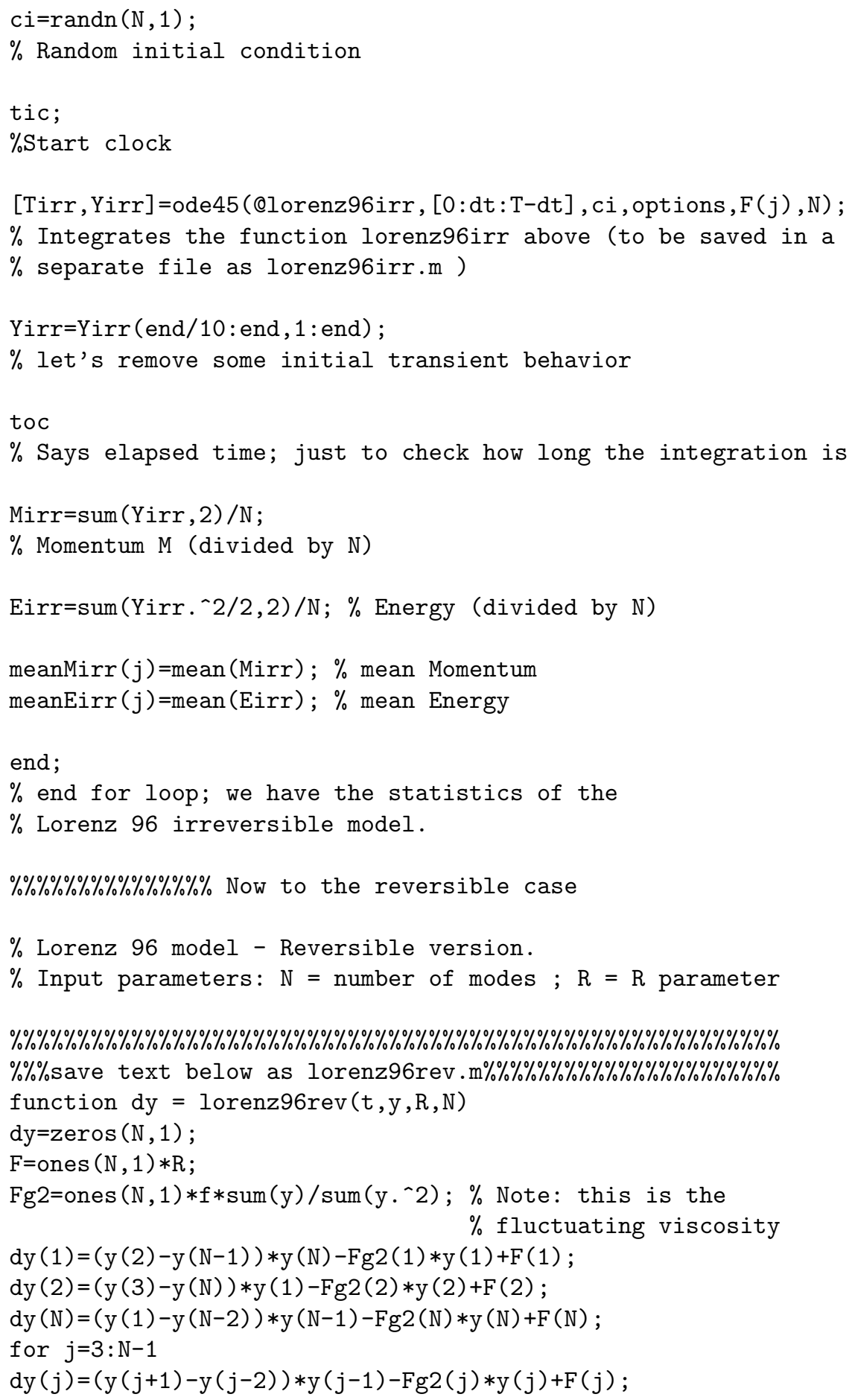




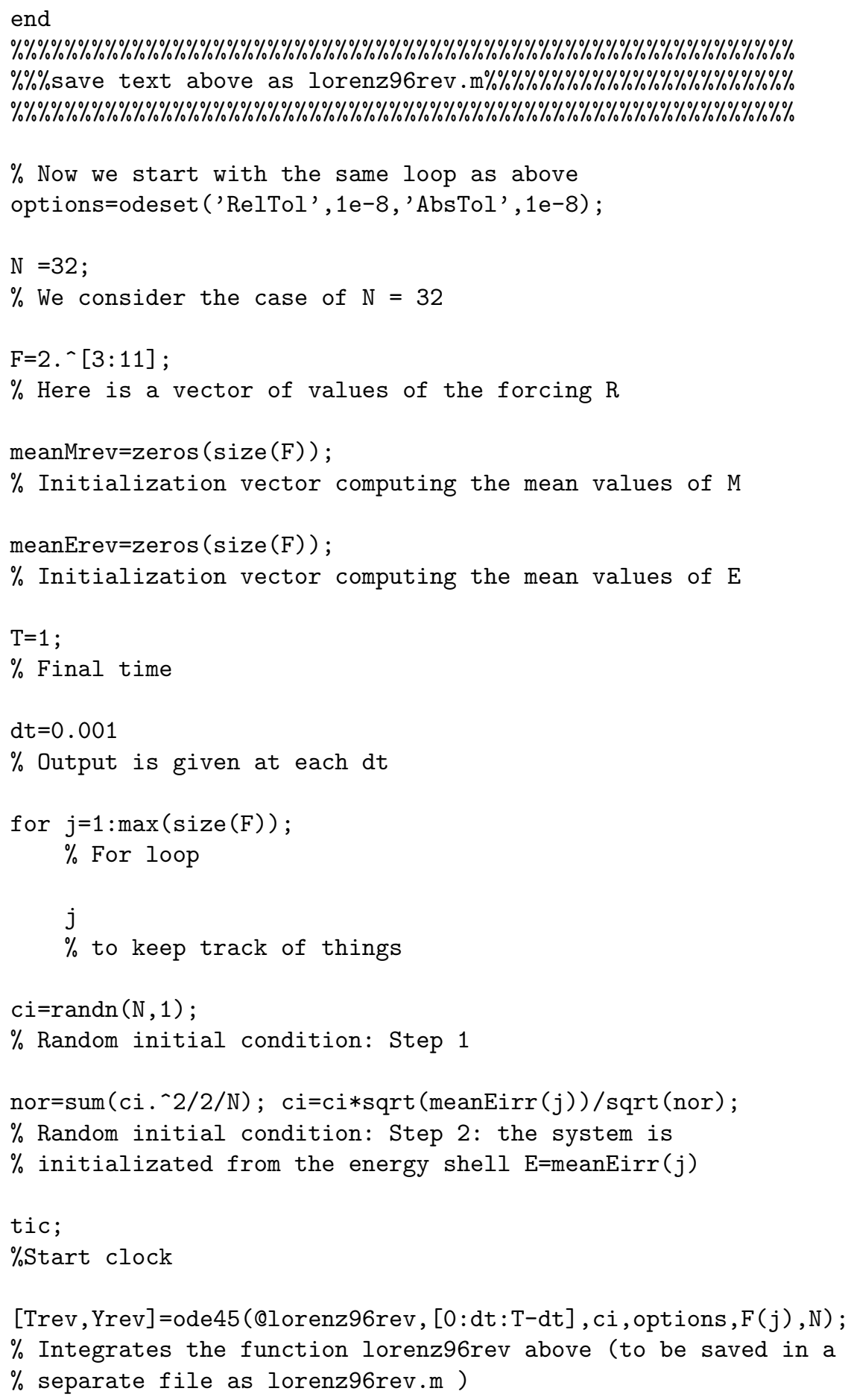


Yrev=Yrev (end/10: end, 1 : end);

$\%$ let's remove some initial transient behavior; not necessary

toc

$\%$ Says elapsed time; just to check how long the integration is

Mrev=sum $($ Yrev , 2) /N ;

$\%$ Momentum M (divided by $N$ )

Erev=sum (Yrev . ^2/2,2)/N;

$\%$ Energy (divided by $N$ ): note: the energy is constant

$\%$ to a very high degree of accuracy (controlled by options)

$\operatorname{meanMrev}(j)=$ mean $($ Mrev) ;

$\%$ mean Momentum

$\operatorname{meanErev}(j)=$ mean $($ Erev) ;

$\%$ mean Momentum

end ;

$\%$ end for loop; we have the statistics of the

\% Lorenz 96 reversible model. Go to Part 2 in Appendix B 


\section{B Script - Part 2: Computing the Lyapunov ex- ponents for the Lorenz 96 irreversible and re- versible models}

\section{$\% \% \% \% \% \% \% \% \% \% \% \%$}

$\% \% \%$ PART 2: Computing the Lyapunov Exponents from the irreversible $\% \% \%$ and reversible models

$\% \% \%$

$\% \% \% \% \% \% \% \%$ Script by V. Lucarini (valerio.lucarini@uni-hamburg.de) $\% \% \% \% \% \% \% \%$ Can be freely distributed according to GNU license.

$\% \% \% \% \% \% \%$ Companion Material to the paper $\% \% \% \% \% \% \%$ Equivalence of Non-equilibrium Ensembles, and $\% \% \% \% \% \% \%$ Representation of Friction in Turbulent Flows: $\% \% \% \% \% \% \%$ the Lorenz 96 model, by G.

$\% \% \% \% \% \% \%$ Gallavotti and V. Lucarini, 2014

$\% \% \% \% \% \% \%$ values given below for the parameters

$\% \% \% \% \% \% \%$ must be checked/optimized by the user

\% Lorenz 96 extended model (trajectory and Jacobian) - Irreversible $\%$ version. Input parameters: $\mathrm{N}=$ numbers of modes ; $\mathrm{R}=\mathrm{R}$ parameter $\% \% \% \% \% \% \% \% \% \% \% \% \% \% \% \% \% \% \% \% \% \% \% \% \% \% \% \% \% \% \% \% \% \% \% \% \% \% \% \% \% \% \% \% \% \% \% \% \% \% \% \% \% \% \% \% \% \% \% \% \% \% \% \% \% \% \%$ $\% \% \%$ save text below as lorenz_ext96irr.m $\% \% \% \% \% \% \% \% \% \% \% \% \% \% \% \% \% \% \% \% \% \% \% \% \% \% \%$ function dy=lorenz_ext96irr $(t, y, R, N)$

$\% \% \% \% \% \%$ Same as lorenz96irr

dy $=\operatorname{zeros}(N *(N+1), 1) ; \quad \%$ a column vector $\mathrm{F}=$ ones $(\mathrm{N}, 1) * \mathrm{R}$;

$\mathrm{dy}(1)=(\mathrm{y}(2)-\mathrm{y}(\mathrm{N}-1)) * \mathrm{y}(\mathrm{N})-\mathrm{y}(1)+\mathrm{F}(1)$;

$\operatorname{dy}(2)=(\mathrm{y}(3)-\mathrm{y}(\mathrm{N})) * \mathrm{y}(1)-\mathrm{y}(2)+\mathrm{F}(2)$;

$\operatorname{dy}(\mathrm{N})=(\mathrm{y}(1)-\mathrm{y}(\mathrm{N}-2)) * \mathrm{y}(\mathrm{N}-1)-\mathrm{y}(\mathrm{N})+\mathrm{F}(\mathrm{N})$;

for $j=3: N-1$

$\operatorname{dy}(j)=(y(j+1)-y(j-2)) * y(j-1)-y(j)+F(j)$;

end

$\% \%$ Initializing the Jacobian

$\mathrm{Y}=\operatorname{Zeros}(\mathrm{N}, \mathrm{N})$;

for $j=1: N$;

for $k=1: N$;

$\mathrm{Y}(\mathrm{j}, \mathrm{k})=\mathrm{y}(\mathrm{N}+\mathrm{j}+(\mathrm{k}-1) * \mathrm{~N})$; 


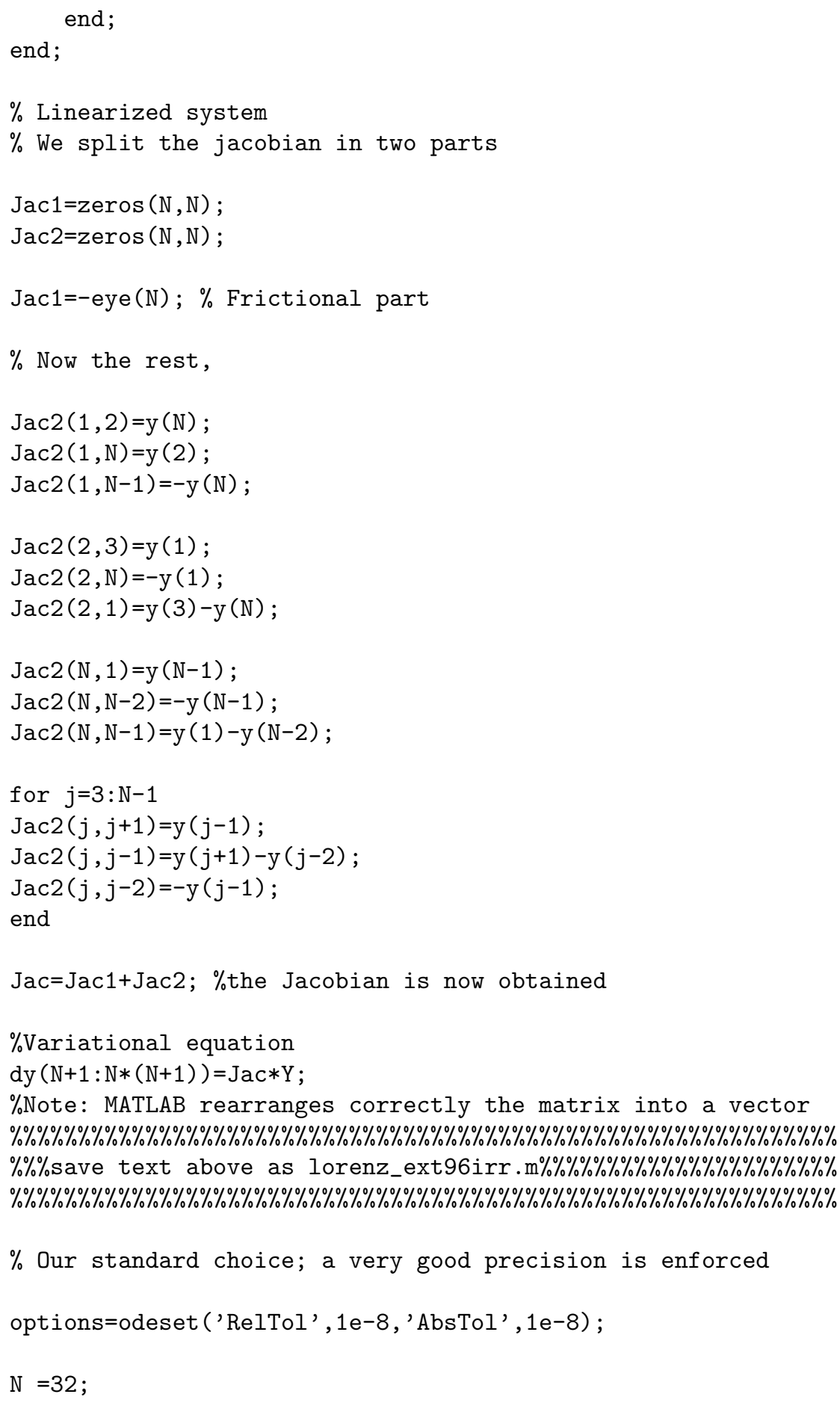


$\%$ We consider the case of $\mathrm{N}=32$

$\mathrm{F}=2 .^{\wedge}[3: 11]$;

$\%$ Here is a vector of values of the forcing $R$

meanLyap=zeros $(\max (\operatorname{size}(F)), N)$;

$\%$ Initialization matrix containing our estimates of the

$\%$ Lyapunov exponents

$\mathrm{T}=100$;

$\%$ Final time; Attention, may need to adapt it to values of $R$

$\%$ because integrations are more and more expensive as $\mathrm{R}$ grows

$d t=0.1$

$\%$ Step of the Grahm-Schmidt orthogonalization procedure;

$\%$ need to adapt is because for large $\mathrm{R}$ lower values are needed

$\%$ or we have no convergence. If so, please define a dt

$\%$ changing with $j$

$\mathrm{n}=1$

$\%$ We get an estimate of Lyapunov exponents every n GS

$\%$ orthogonalizations.

for $j=1: \max (\operatorname{size}(F))$;

$\%$ For loop

$j$

$\%$ to keep track of things

$\mathrm{Ci}=\operatorname{randn}(\mathrm{N}, 1)$;

$\%$ Random initial condition

tic; \%Start clock

[Texpirr, Lexpirr] =lyapunov_mod (N, @lorenz_ext96irr, @ode45, ।

$0, d t, T, c i, n, o p t i o n s, F(j), N)$;

$\%$ Computes the Lyapunov exponents for the Lorenz 96 irreversible

$\%$ model calling the function lorenz_ext96irr above (to be saved in a $\%$ separate file as lorenz_ext96irr.m )

$\%$ We use a slightly modified version of the freely available $\%$ lyapunov.m function due to Govorukhin V.N. (2004) see references.

$\%$ We accomodate for the possibily of reading two parameters ( $F$ and $R$ $\%$ in our case). The Benettin et al. (1980) algorithm is used.

$\%$ The routine lyapunov_mod.m is available on request 


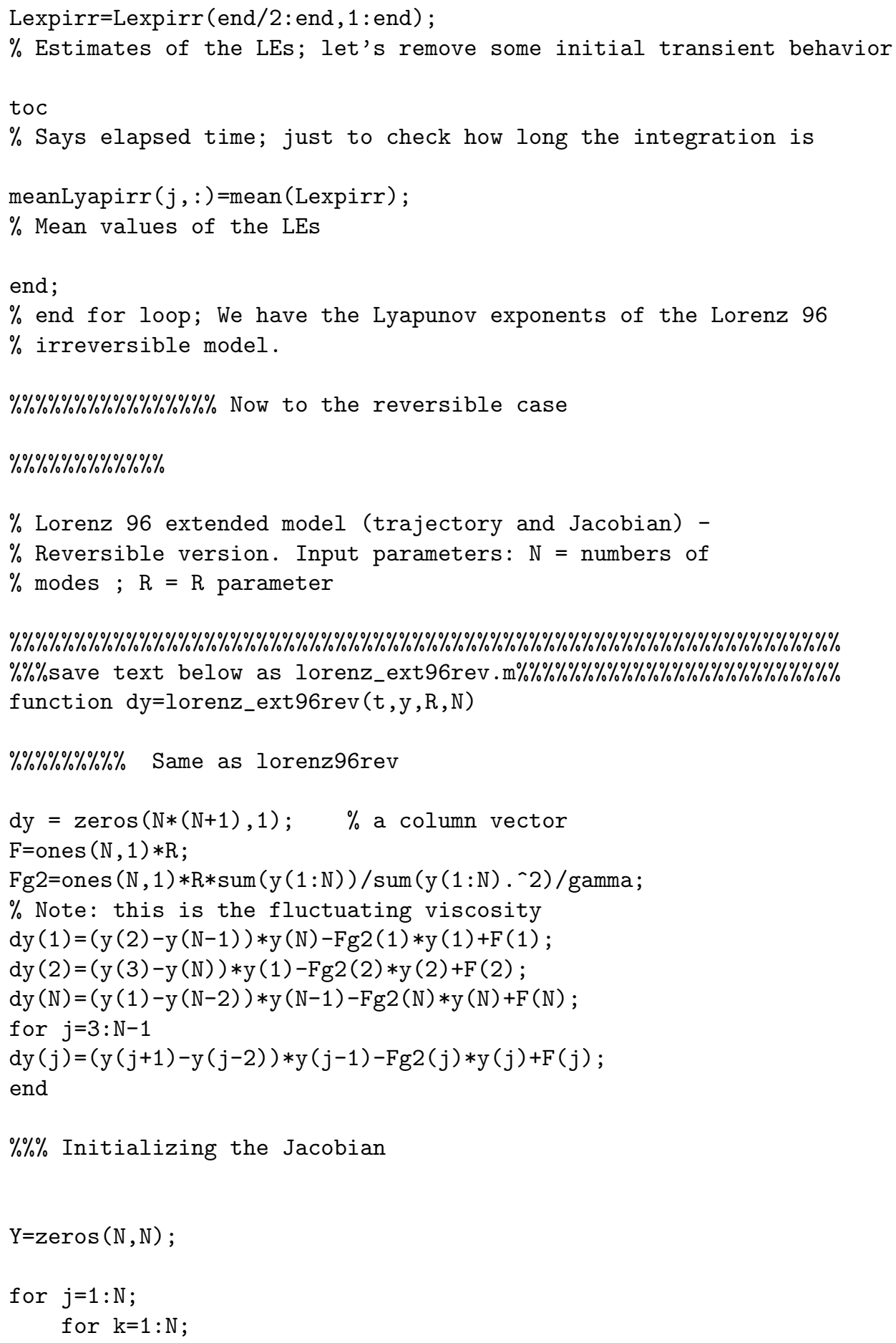




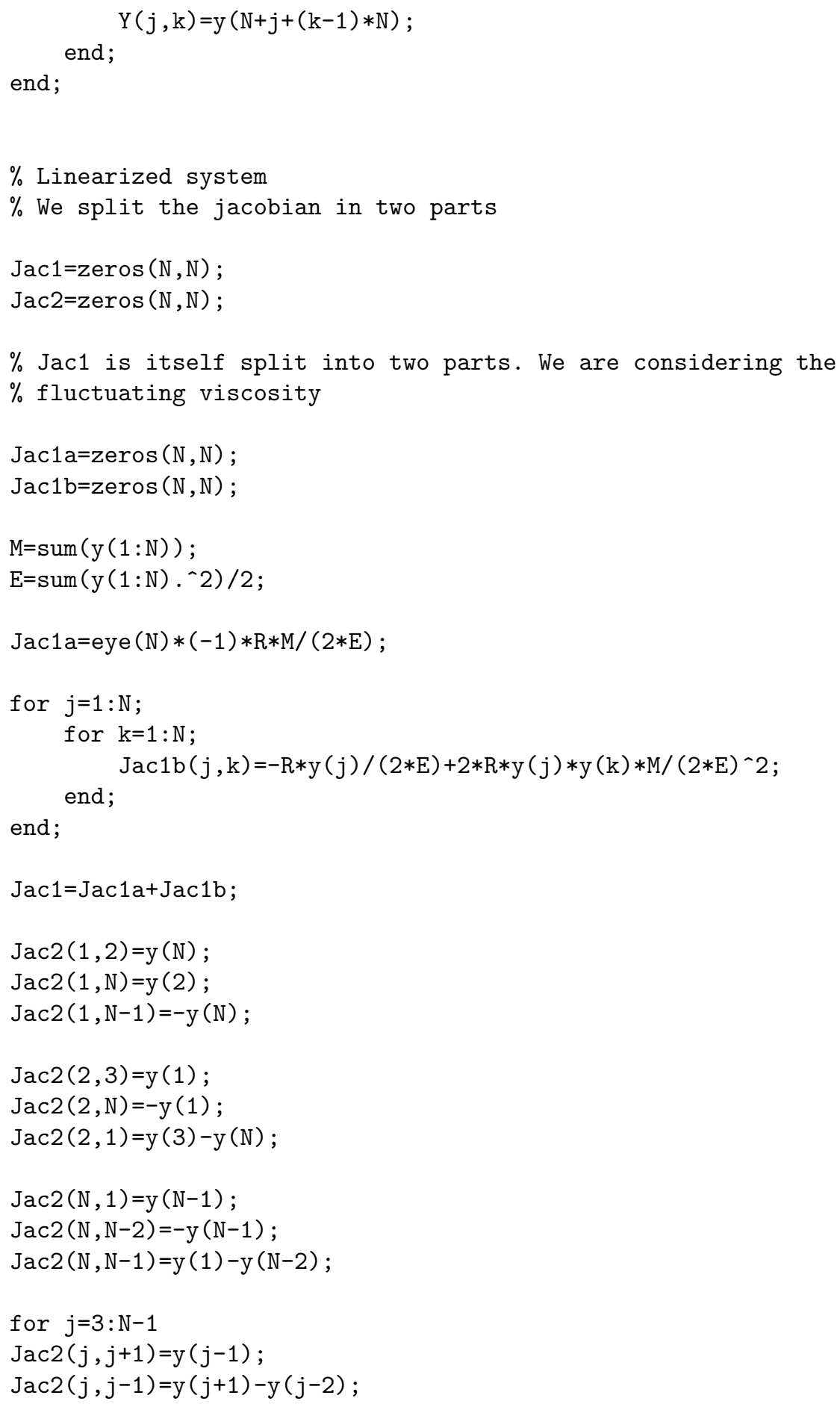




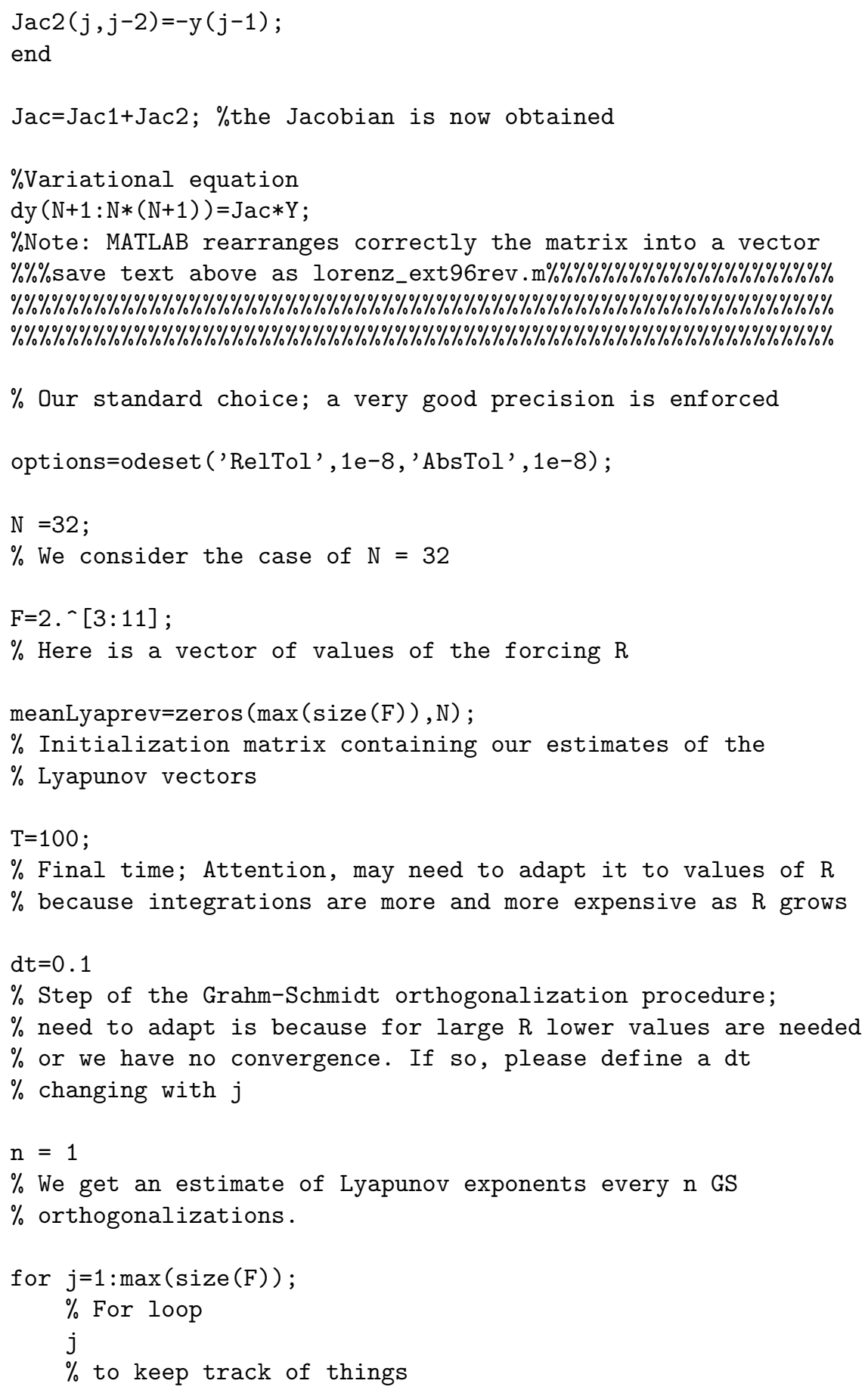




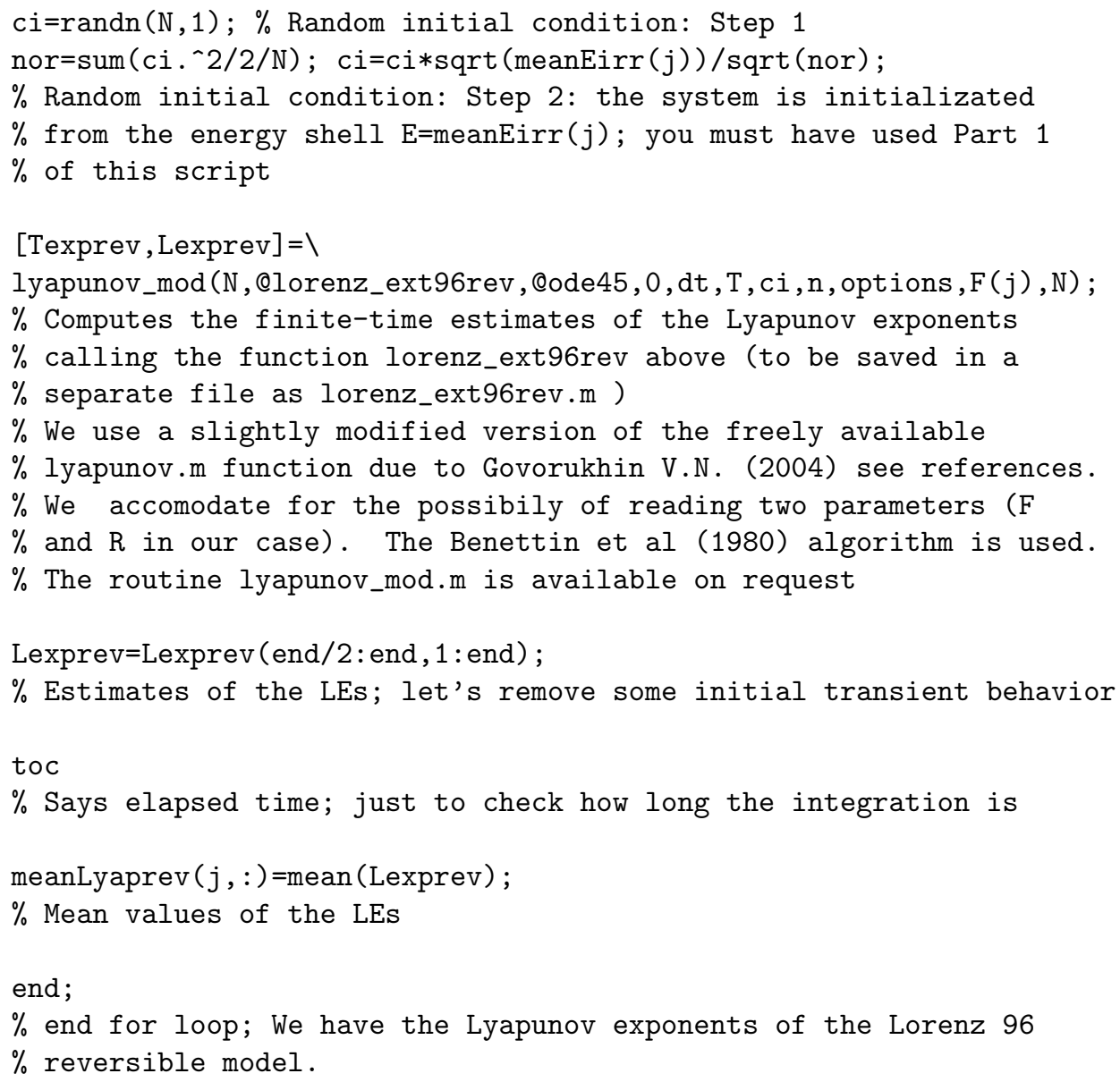




\section{References}

[1] R. V. Abramov and A. Majda. New approximations and tests of linear fluctuation-response for chaotic nonlinear forced-dissipative dynamical systems. Journal of Nonlinear Science, 18:303-341, 2008. 10.1007/s00332-0079011-9.

[2] G. Benettin, L. Galgani, A. Giorgilli, and J. M. Strelcyn. Lyapunov characteristic exponents for smooth dynamical systems and for Hamiltonian systems; a method for computing all of them. Part 1: Theory. Meccanica, 15(1):9-20, 1980.

[3] R. Blender, J. Wouters, and V. Lucarini. Avalanches, breathers, and flow reversal in a continuous lorenz-96 model. Phys. Rev. E, 88:013201, Jul 2013.

[4] A.W. Bowman and A. Azzalini. Applied Smoothing Techniques for Data Analysis. Oxford University Press, Oxford, 1997.

[5] C. Dettman and G. Morriss. Proof of conjugate pairing for an isokinetic thermostat. Physical Review E, 53:5545-5549, 1996.

[6] U. Dressler. Symmetry property of the lyapunov exponents of a class of dissipative dynamical systems with viscous damping. Physical Review A, 38:21032109, 1988.

[7] P. Sagaut E. Garnier, N. Adams. Large Eddy Simulation for compressible flows. Springer, New York, 2009.

[8] J.-P. Eckmann and D. Ruelle. Ergodic theory of chaos and strange attractors. Rev. Mod. Phys., 57:617655, 1985.

[9] G. Gallavotti. Reversible Anosov diffeomorphisms and large deviations. Mathematical Physics Electronic Journal (MPEJ), 1:1-12, 1995.

[10] G. Gallavotti. Extension of Onsager's reciprocity to large fields and the chaotic hypothesis. Physical Review Letters, 77:4334-4337, 1996.

[11] G. Gallavotti. Dynamical ensembles equivalence in fluid mechanics. Physica D, 105:163-184, 1997.

[12] G. Gallavotti. Breakdown and regeneration of time reversal symmetry in nonequilibrium statistical mechanics. Physica D, 112:250-257, 1998.

[13] G. Gallavotti. Fluctuations and entropy driven space-time intermittency in Navier-Stokes fluids, in Mathematical Physics 2000, Ed. E. Fokas, A. Grigoryan, T. Kibble, B. Zegarlinski. World Scientific, London, 2000.

[14] G. Gallavotti. Non equilibrium in statistical and fluid mechanics. ensembles and their equivalence. entropy driven intermittency. Journal of Mathematical Physics, 41:4061-4081, 2000.

[15] G. Gallavotti. Foundations of Fluid Dynamics. (second printing) Springer Verlag, Berlin, 2005. 
[16] G. Gallavotti. Microscopic chaos and macroscopic entropy in fluids. Journal of Statistical Mechanics (JSTAT), 2006:P10011 (+9), 2006.

[17] G. Gallavotti. Aspects of Lagrange's Mechanics and their Legacy. arXiv:1305.3438, pages 1-23, 2013.

[18] G. Gallavotti, F. Bonetto, and G. Gentile. Aspects of the ergodic, qualitative and statistical theory of motion. Springer Verlag, Berlin, 2004.

[19] G. Gallavotti and E. G. D. Cohen. Dynamical ensembles in nonequilibrium statistical mechanics. Physical Review Letters, 74:2694-2697, 1995.

[20] G. Gallavotti and E. G. D. Cohen. Dynamical ensembles in stationary states. Journal of Statistical Physics, 80:931-970, 1995.

[21] G. Gallavotti, L. Rondoni, and E. Segre. Lyapunov spectra and nonequilibrium ensembles equivalence in 2d fluid. Physica D, 187:358-369, 2004.

[22] Sarah Hallerberg, Diego Pazo, Juan M. Lopez, and Miguel A. Rodriguez. Logarithmic bred vectors in spatiotemporal chaos: Structure and growth. Phys. Rev. E, 81:066204, Jun 2010.

[23] A. Karimi and M. R. Paul. Extensive chaos in the lorenz-96 model. Chaos: An Interdisciplinary Journal of Nonlinear Science, 20(4):-, 2010.

[24] R. Livi, A. Politi, and S. Ruffo. Distribution of characteristic exponents in the thermodynamic limit. Journal of Physics A, 19:2033-2040, 1986.

[25] E. Lorenz. Deterministic non periodic flow. Journal of the Atmospheric Science, 20:130-141, 1963.

[26] E. Lorenz. Designing chaotic models. Journal of the Atmospheric Science, 62:1574-1587, 2005.

[27] E. Lorenz and K. Emanuel. Optimal Sites for Supplementary Weather Observations: Simulation with a Small Model. Journal of the Atmospheric Science, 55:399-414, 1998.

[28] V. Lucarini. Response theory for equilibrium and non-equilibrium statistical mechanics: causality and generalized kramers-kronig relations. J. Stat. Phys., 131(3):543-558, 2008.

[29] V. Lucarini. Evidence of dispersion relations for the nonlinear response of lorenz 63 system. J. Stat. Phys., 134:38140, 2009.

[30] V. Lucarini, R. Blender, C. Herbert, S. Pascale, F. Ragone, and J. Wouters. Mathematical and Physical Ideas for Climate Science. ArXiv e-prints, November 2013.

[31] V. Lucarini and S. Sarno. A Statistical Mechanical Approach for the Computation of the Climatic Response to General Forcings . Nonlin. Processes Geophys, 18:7-28, 2011. 
[32] D. Orrell. Model error and predictability over different timescales in the lorenz '96 systems. Journal of the Atmospheric Sciences, 60(17):2219-2228, 2014/03/24 2003.

[33] Stephen Pope. Turbulent Flows. Cambridge University Press, Cambrdge, 2000.

[34] F. Ragone, V. Lucarini, and F. Lunkeit. A new framework for climate sensitivity and prediction. ArXiv e-prints, March 2014.

[35] D. Ruelle. Chaotic Evolution and Strange Attractors. Cambridge University Press, 1989.

[36] D. Ruelle. General linear response formula in statistical mechanics, and the fluctuation-dissipation theorem far from equilibrium. Phys. Letters A, 245:220$224,1998$.

[37] D. Ruelle. A review of linear response theory for general differentiable dynamical systems. Nonlinearity, 22:855-870, 2009.

[38] D. Ruelle and F. Takens. On the nature of turbulence. Communications in Mathematical Physics, 20:167-192, 1971.

[39] Pierre Sagaut. Large eddy simulation for incompressible flows. Springer, New York, 2006.

[40] Z.S. She and E. Jackson. Constrained Euler system for Navier Stokes turbulence. Phys. Rev. Lett., 70(9):1255-1258, 1993.

[41] Ya. G. Sinai. Lectures in ergodic theory. Lecture notes in Mathematics. Princeton University Press, Princeton, 1977.

[42] Joseph Smagorinsky. Large eddy simulation of complex engineering and geophysical flows. In B. Galperin and S. A. Orszag, editors, Evolution of Physical Oceanography, pages 3-36. Cambridge University Press, 1993.

[43] Anna Trevisan, Massimo D'Isidoro, and Olivier Talagrand. Four-dimensional variational assimilation in the unstable subspace and the optimal subspace dimension. Quarterly Journal of the Royal Meteorological Society, 136(647):487496, 2010.

[44] Anna Trevisan and Francesco Uboldi. Assimilation of standard and targeted observations within the unstable subspace of the observation-analysis-forecast cycle system. Journal of the Atmospheric Sciences, 61(1):103-113, 2014/03/24 2004.

[45] Daniel S. Wilks. Effects of stochastic parametrizations in the lorenz '96 system. Quarterly Journal of the Royal Meteorological Society, 131(606):389-407, 2005. 